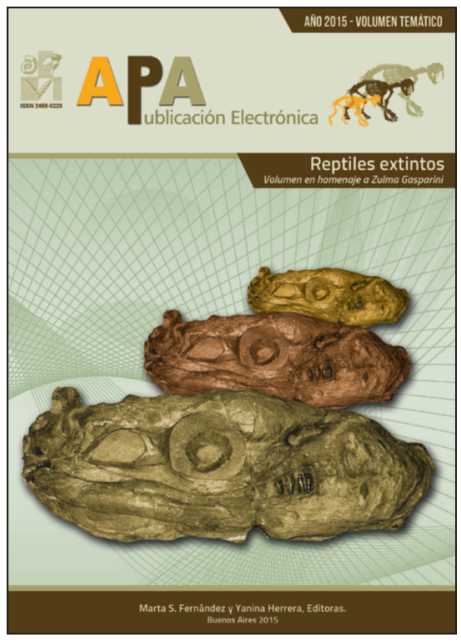

\title{
EL REGISTRO DE SAUROPODOMORPHA (DINOSAURIA) DE LA ARGENTINA
}

\section{ALEJANDRO OTERO 1}

LEONARDO SALGADO²

${ }^{1}$ CONICET. División Paleontología Vertebrados, Museo de La Plata. Paseo del Bosque s/n, B1900FWA La Plata, Argentina.

${ }^{2}$ CONICET. Instituto de Investigación en Paleobiología y Geología, Universidad Nacional de Río Negro. Av. General Roca 1242, R8332EXZ General Roca, Río Negro, Argentina.

Recibido: 4 de Junio de 2015 - Aceptado: 12 de Agosto de 2015

Para citar este artículo: Alejandro Otero y Leonardo Salgado (2015). El registro de Sauropodomorpha (Dinosauria) de la Argentina. En: M. Fernández y Y. Herrera (Eds.) Reptiles Extintos - Volumen en Homenaje a Zulma Gasparini. Publicación Electrónica de la Asociación Paleontológica Argentina 15(1): 69-89.

Link a este artículo: http://dx.doi.org/10.5710/PEAPA.04.06.2015.100

DESPLAZARSE HACIA ABAJO PARA ACCEDER AL ARTÍ́CULO

Asociación Paleontológica Argentina Maipú $6451^{\circ}$ piso, C1006ACG, Buenos Aires República Argentina

Tel/Fax (54-11) 4326-7563 Web: www.apaleontologica.org.ar

Otros artículos en Publicación Electrónica de la APA 15(1):

\section{de la Fuente \& Sterli}

ESTADO DEL CONOCIMIENTO DE LAS TORTUGAS EXTINTAS DEL TERRITORIO ARGENTINO: UNA PERSPECTIVA HISTÓRICA.

\section{Paulina Carabajal}

GUIA PARA EL ESTUDIO DE LA NEUROANATOMÍA DE DINOSAURIOS SAURISCHIA, CON ENFASIS EN FORMAS SUDAMERICANAS.

\section{Pol \& Leardi}

DIVERSITY PATTERNS OF NOTOSUCHIA (CROCODYLIFORMES, MESOEUCROCODYLIA) DURING THE CRETACEOUS OF GONDWANA. 


\title{
EL REGISTRO DE SAUROPODOMORPHA (DINOSAURIA) DE LA ARGENTINA
}

\author{
ALEJANDRO OTERO' 1 Y LEONARDO SALGADO²
}

${ }^{1}$ CONICET. División Paleontología Vertebrados, Museo de La Plata. Paseo del Bosque s/n, B1900FWA La Plata, Argentina. alexandros.otero@gmail.com ${ }^{2}$ CONICET. Instituto de Investigación en Paleobiología y Geología, Universidad Nacional de Río Negro. Av. General Roca 1242, R8332EXZ General Roca, Río Negro, Argentina.Isalgado@unrn.edu.ar

\begin{abstract}
Resumen. Sauropodomorpha comprende a los dinosaurios herbívoros más abundantes y diversos, con un registro global. En la Argentina su registro es particularmente rico y abundante y sus taxones han dado luz a los hitos más importantes de la historia evolutiva de este grupo de dinosaurios saurisquios. El origen de Sauropodomorpha, la transición hacia Sauropoda, así como la diversificación de Diplodocoidea y Macronaria son, en gran medida, ejemplificados por el registro proveniente de las capas mesozoicas de la Argentina. En esta contribución se presenta el registro actualizado de los géneros válidos de Sauropodomorpha de la Argentina, incluyendo datos sobre la procedencia geográfica y estratigráfica y relaciones filogenéticas.
\end{abstract}

Palabras clave. Sauropodomorpha basales. Sauropoda. Diplodocoidea. Macronaria. Argentina. Registro fósil.

Abstract. THE RECORD OF SAUROPODOMORPHA (DINOSAURIA) OF ARGENTINA. Sauropodomorpha includes the most abundant and diverse herbivorous dinosaurs, with a worldwide record. In Argentina, its record is particularly rich and abundant, and its taxa have shed light to the most important milestones in the evolutionary history of this group of saurischian dinosaurs. The origin of Sauropodomorpha, the transition to Sauropoda and the diversification of Diplodocoidea and Macronaria are largely exemplified by the Mesozoic record from Argentina. In this contribution the updated record of the Sauropodomorpha genera of Argentina, including data on the geographic and stratigraphic record, origin and phylogenetic relationships, is presented.

Key words. Basal Sauropodomorpha. Sauropoda. Diplodocoidea. Macronaria. Argentina. Fossil record.

SAUROPODOMORPHa Huene, 1932 es uno de los grupos de dinosaurios más exitosos que habitaron todos los continentes, desde el Triásico Tardío (Carniense, aproximadamente $225 \mathrm{Ma}$ ) hasta el Cretácico Tardío (Maastrichtiense, $64 \mathrm{Ma}$ ) (Galton y Upchurch, 2004; Smith y Pol, 2007; Cerda et al., 2012). Comprenden a un grupo basal, tradicionalmente denominado "prosaurópodos", y a los Sauropoda Marsh, 1878. Los sauropodomorfos basales fueron de pequeño a mediano tamaño, con largos cuellos y cráneos reducidos, mayormente bípedos (aunque algunas formas fueron también cuadrúpedas) que dominaron los ambientes continentales desde el Triásico Tardío hasta el Jurásico Temprano (Galton y Upchurch, 2004). "Prosauropoda" es un grupo tradicionalmente considerado como monofilético (Galton, 1990; Galton y Upchurch, 2004; Upchurch et al., 2007). Sin embargo, en los últimos diez años, nuevos descubrimientos, redes- cripciones de taxones ya conocidos y la aparición de nuevas y mejoradas matrices de datos filogenéticos, arrojaron nueva luz sobre este grupo, que pasó a considerarse como una asociación parafilética de sucesivos grupos hermanos a Sauropoda (Yates, 2007a; Apaldetti et al., 2012; Otero y Pol, 2013) (Fig. 1.1). De esta manera, el término "Prosauropoda" cayó en desuso, siendo reemplazado por "Sauropodomorpha basales", los cuales básicamente incluyen a todos los dinosaurios sauropodomorfos que se encuentran por fuera del clado Sauropoda. Más allá de su naturaleza parafilética, las relaciones internas de este grupo se encuentran relativamente bien establecidas. En este sentido, las ramas más basales de este grupo se encuentran bien representadas por el registro sudamericano de Eoraptor lunensis Sereno, Rogers y Monetta, 1993, Saturnalia tupiniquim Langer, Abdala, Richter y Benton, 1999, Chromogisaurus novasi Ezcurra, 
2010, Panphagia protos Martínez y Alcober, 2009, y Pampadromaeus barberanai Cabreira, Schultz, Bittencourt, Soares, Fortier, Silva y Langer, 2011, como los actores principales; el "núcleo" de los sauropodomorfos basales compuesto por los monofiléticos Plateosauridae y Massospondylidae (Galton y Upchurch, 2004; Apaldetti et al., 2011, 2012), mientras que un creciente número de sauropodomorfos se ubican de manera sucesiva como grupos externos a Sauropoda (Pol et al., 2011; Otero y Pol, 2013; Otero et al., 2015).

Sauropodomorpha fue definido por Salgado et al. (1997a) como "el clado que incluye al ancestro común más reciente de Prosauropoda y Sauropoda y a todos sus descendientes". Esta definición considera a ambos grupos de sauropodomorfos como monofiléticos, permitiendo una definición basada en el nodo. Teniendo en cuenta la naturaleza parafilética de las formas basales de Sauropodomorpha, es preferible una definición basada en la rama: "el clado más inclusivo que contiene a Saltasaurus Bonaparte y Powell, 1980 pero no a Passer o Triceratops Marsh, 1889" (Sereno, 2007).
1

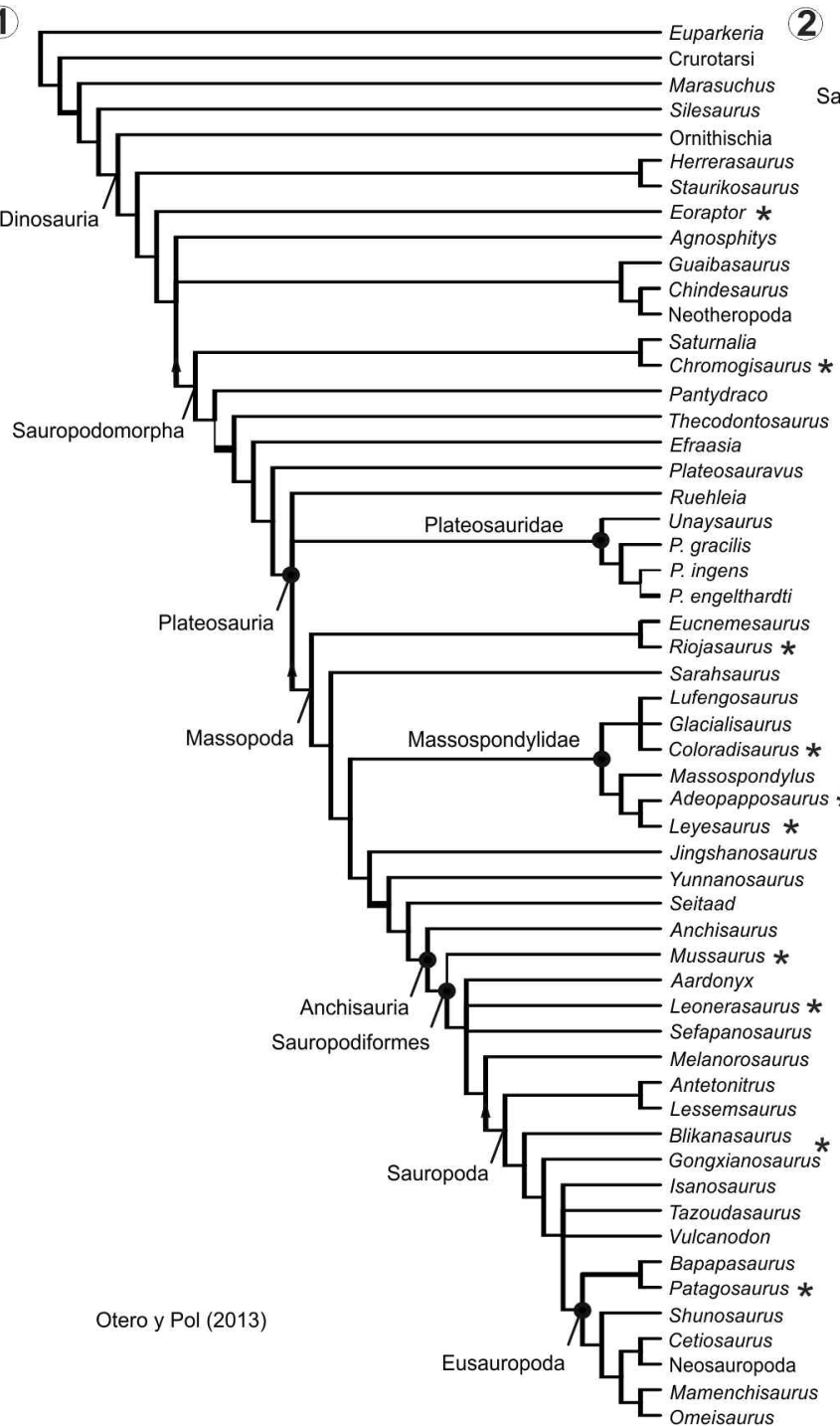

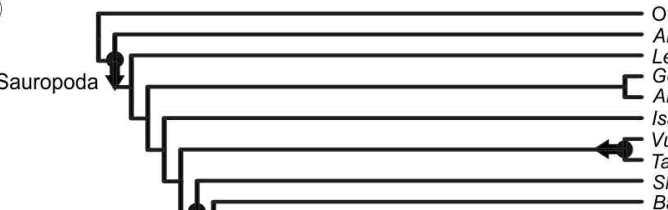
tetonitrus ssemsaurus * Amygdalodon *
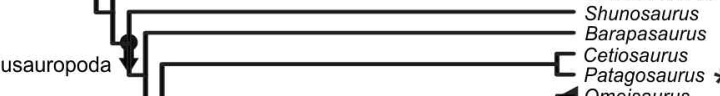
Patagosaurus Mameisaurus
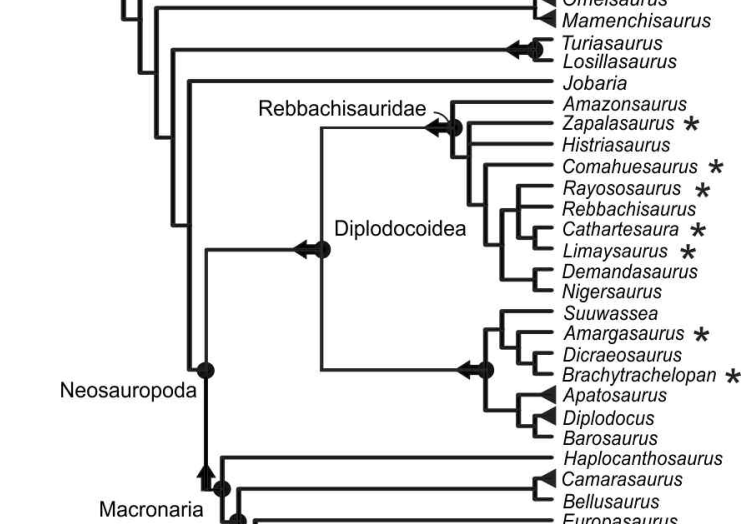
Camarasauromorpha 1 Bellusaurus

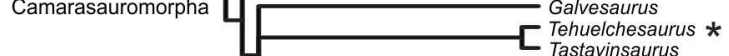
- Tastavinsaurus - Euhelopus

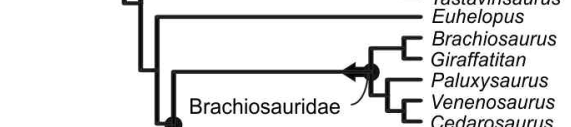
Titanosauriformes Venenosaurus Erketu

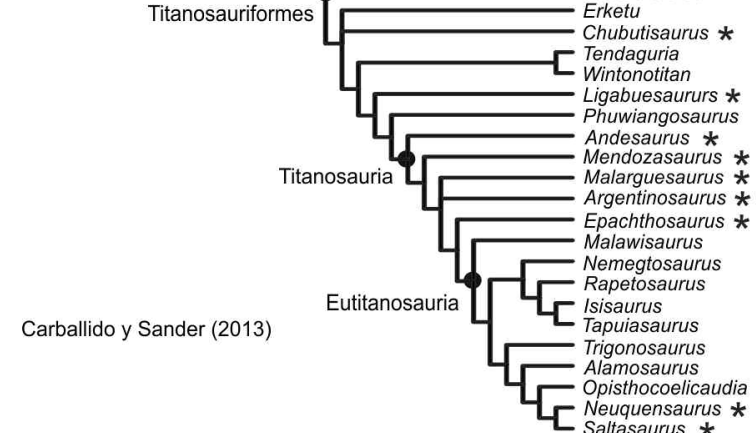

Figura 1. 1, Relaciones filogenéticas de los Sauropodomorpha basales. 2, Relaciones filogenéticas de Sauropoda. Los asteriscos denotan los sauropodomorfos con registro en Argentina. 
Sauropoda es definido como el clado más inclusivo que contiene a Saltasaurus pero no a Melanorosaurus Haughton, 1924 (Yates, 2007b). Con aproximadamente 150 especies válidas y una distribución mundial, Sauropoda comprende a los dinosaurios herbívoros dominantes desde el Jurásico Medio hasta el Cretácico Tardío. Su plan corporal único, caracterizado por su gigantesco tamaño, locomoción graviportal, largos cuello y cola, y cráneos reducidos, hicieron de este grupo un ícono indiscutido en la cultura popular, desde los comienzos de los hallazgos (Sanz, 2007; Otero y Gasparini, 2014).

Hasta la fecha, la filogenia de jerarquías altas de los saurópodos está relativamente bien establecida, compuesta por Sauropoda basales (e.g., Lessemsaurus Bonaparte, 1999, del Triásico Superior de la Formación Los Colorados, provincia de La Rioja) y Eusauropoda (e.g., Patagosaurus Bonaparte, 1979, del Jurásico Medio de Cerro Cóndor, provincia del Chubut), registradas en el Jurásico Inferior y Medio, una radiación de Neosauropoda, compuesta por Diplodocoidea (e.g., Amargasaurus Salgado y Bonaparte, 1991, del Cretácico Inferior de la provincia del Neuquén) y Macronaria (e.g., titanosaurios y formas afines), del Jurásico Medio al Cretácico Superior (Carballido y Sander, 2013) (Fig. 1.2).

La Argentina ha sido un importante foco de descubrimientos y estudios en sauropodomorfos desde fines del siglo XIX a la fecha (e.g., Salgado y Bonaparte, 2007). La presencia de abundantes afloramientos de rocas mesozoicas, así como también el estudio y la investigación constante llevada a cabo durante los últimos 40 años, ubican a la Argentina entre los centros más importantes a nivel mundial (Fig. 2).

En esta contribución ponemos al día el listado del registro de dinosaurios sauropodomorfos conocidos en la Argentina desde una perspectiva histórica, incluyendo los primeros descubrimientos y estudios, los actores principales, así como también las diferentes líneas de investigación que se están llevando a cabo actualmente.

Abreviaturas institucionales. MACN, Museo Argentino de Ciencias Naturales "Bernardino Rivadavia", Ciudad Autónoma de Buenos Aires, Argentina; MMCH, Museo Municipal "Ernesto Bachmann", Villa El Chocón, Neuquén, Argentina; PVL, Instituto "Miguel Lillo", San Miguel de Tucumán, Tucumán, Argentina.

\section{RESEÑA DE LOS PRINCIPALES GRUPOS DE SAUROPODOMORPHA \\ Sauropodomorpha basales (Anexo 1)}

La ocurrencia de sauropodomorfos basales en la Argentina se conoce desde la década de los sesenta, a partir de los trabajos pioneros de los paleontólogos argentinos Rodolfo Casamiquela y José Bonaparte (ver más abajo). Sin embargo, el registro más antiguo del grupo se remonta al año 1993, con el hallazgo de Eoraptor lunensis, de la Formación Ischigualasto (Carniense/Noriense, Triásico Superior; Martínez et al., 2011), de la provincia de San Juan. Eoraptor fue inicialmente considerado un terópodo basal y esas afinidades permanecieron por un largo período de tiempo (Novas, 1994; Sereno, 1999, 2006; Tykoski, 2005; Ezcurra, 2010); incluso fue considerado como un saurisquio basal por fuera de Theropoda y Sauropodomorpha (Langer, 2004; Brusatte et al., 2010). Luego de una re-preparación del material original, re-codificación e inclusión en matrices filogenéticas con mayor muestreo de taxones y caracteres, Eoraptor es actualmente considerado como un miembro basal de Sauropodomorpha (Martínez et al., 2011; Sereno et al., 2013; Otero et al., 2015 y referencias allí citadas).

La Formación Ischigualasto es la que brindó más taxones basales de este grupo, además de Eoraptor: Panphagia protos y Chromogisaurus novasi. Panphagia es conocido a partir de un esqueleto incompleto, incluyendo material craneano y es actualmente considerado el sauropodomorfo más primitivo, con un mosaico de caracteres de este grupo (e.g., supraoccipital amplio con muescas profundamente marcadas para la vena occipitalis externa y una cresta nucal baja), pero también con caracteres teropodianos (e.g., receso flocular timpánico anterior de gran tamaño) (Martínez et al., 2012). Chromogisaruus es un taxón cercanamente emparentado a Saturnalia, de la Formación Santa Maria, Brasil, y se conoce a partir de un esqueleto incompleto, cuyos caracteres compartidos con el taxón brasileño permiten reconocer una subfamilia propia, Saturnaliinae (Ezcurra, 2010).

La provincia de La Rioja también ha contribuido con una rica fauna de sauropodomorfos. Bonaparte reportó la presencia de Riojasaurus incertus Bonaparte, 1967, del Triásico Superior de la Formación Los Colorados (Bonaparte, 1967, 1971), referido originalmente como un "prosaurópodo" melanorosáurido, aunque menos robusto que las formas sudafri- 

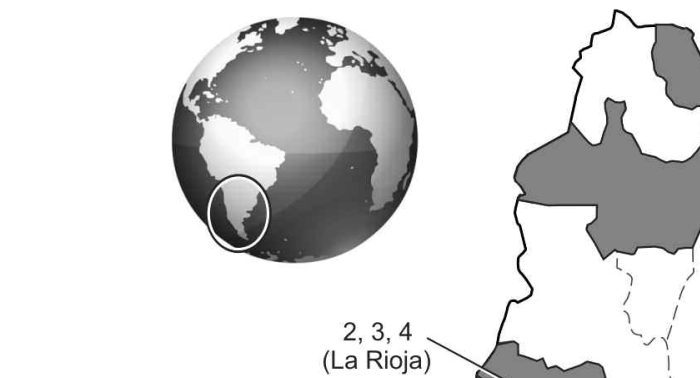

$11,12,13,14,15,16,17$ $18,19,20,21,22,23,24$ $25,26,27,28,29,30$ (Neuquén)

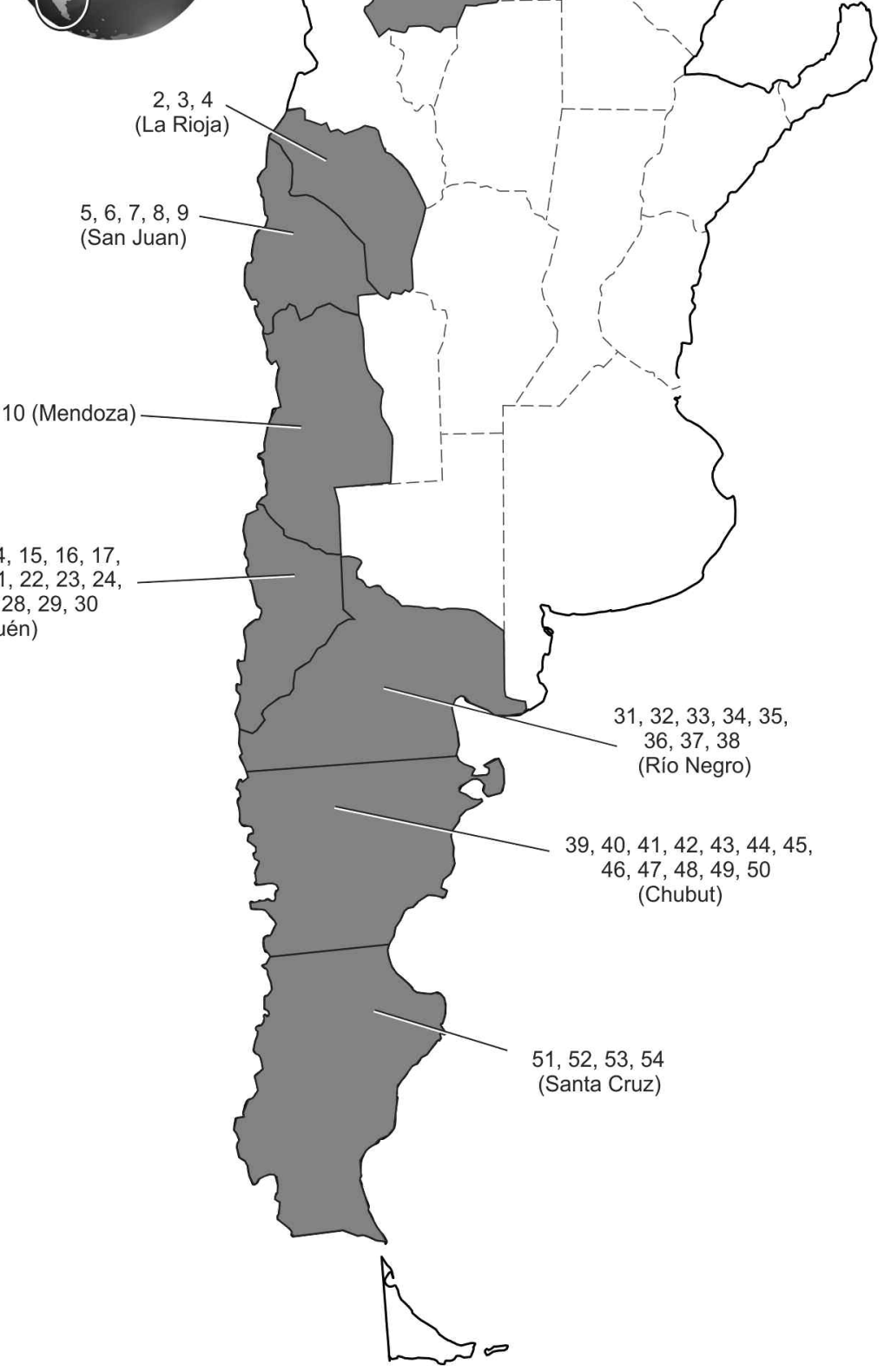

1- Saltasaurus loricatus

2- Riojasaurus incertus

3- Coloradisaurus brevis

4- Lessemsaurus sauropoides

5- Leyesaurus marayensis

6- Chromogisaurus novasi

7- Adeopapposaurus mognai

8- Eoraptor lunensis

9- Panphagia protos

10- Mendozasaurus neguyelap

11- Limaysaurus tessonei

12- Rayososaurus agrioensis

13- Amargasaurus cazaui

14- Zapalasaurus bonapartei

15- Comahuesaurus windhauseni

16- Leinkupal laticauda

17- Ligabuesaurus leanzai

18- Agustinia ligabuei

19- Andesaurus delgadoi

20- Argentinosaurus hunculensis

21- Barrosasaurus casamiquelai

22- Muyelensaurus pecheni

23- Pitekunsaurus macayai

24- Narambuenatitan palomoi

25- Overosaurus paradasorum

26- Futalognkosaurus dukei

27 - Rinconsaurus caudamirus

28- Panamericansaurus schroederi

29- Petrobrasaurus puestohernandezi

30- Neuquensaurus australis

31- Cathartesaura anaerobica

32- Pellegrinisaurus powelli

33- Aeolosaurus rionegrinus

34- Antarctosaurus wichmannianus

35- Laplatasaurus araukanicus

36- Bonitasaura salgadoi

37- Bonatitan reigi

38- Rocasaurus muniozi

39- Amygdalodon patagonicus

40- Patagosaurus fariasi

41- Volkheimeria chubutensis

42- Brachytrachelopan mesai

43- Tehuelchesaurus benitez

44- Argyrosaurus superbus

45- Chubutisaurus insignis

46- Katepensaurus goicoecheai

47- Epachthosaurus sciuttoi

48- Aeolosaurus colhuehuapensis

49- Elaltitan lilloi

50- Leonerasaurus taquetrensis

51- Puertasaurus reuilli

52- Drusilasaura deseadensis

53- Dreadgnouthus schrani

54- Mussaurus patagonicus

Figura 2. Ocurrencia de Sauropodomorpha en el territorio argentino. Las flechas no indican el lugar preciso de los hallazgos.

canas coetáneas (Bonaparte, 1971) (Fig. 3). Análisis filogenéticos recientes, sin embargo, muestran a Riojasaurus como un Massopoda basal (i.e., "el clado más inclusivo que contiene a Saltasaurus pero no a Plateosaurus von Mayer, 1837", Yates, 2007b), es decir, más cercano a formas como Massos- pondyus Owen, 1854 que a Melanorosaurus (Upchurch et al., 2007; Yates, 2007a; Otero et al., 2015).

En 1978, Bonaparte presentó otro "prosaurópodo" de la Formación Los Colorados, Coloradisaurus ('Coloradia) brevis Bonaparte, 1978, referido en ese entonces como un Pla- 
teosauridae, dadas las evidentes similitudes con Plateosaurus y Lufengosaurus Young, 1941 (Bonaparte, 1978, p. 329). Nuevas descripciones de Coloradisaurus muestran a este taxón como un miembro de Massospondylidae (Apaldetti et al., 2012, 2014).

Los sauropodomorfos del Jurásico Inferior de la provincia de San Juan están representados por los massospondylidos Adeopapposaurus mognai Martínez, 2009 de la Formación Cañón del Colorado y Leyesaurus marayensis Apaldetti, Martínez, Alcober y Pol, 2011 de la Formación Quebrada del Barro.

La ocurrencia de sauropodomorfos basales en Patagonia tiene una historia que comienza aproximadamente hace medio siglo atrás. Entre 1961 y 1964, el Museo de La Plata y el Instituto "Miguel Lillo" (Tucumán) Ilevaron a cabo una serie de campañas lideradas por Rodolfo Casamiquela (Museo de La Plata) en la provincia de Santa Cruz, a capas de edad Triásico Superior (Formación Laguna Colorada), cerca de la estancia "El Tranquilo". Aquellas campañas sacaron a la luz los primeros hallazgos de sauropodomorfos basales de la Argentina (Casamiquela, 1964). Una década después, se reporta la presencia de un nuevo sauropodomorfo basal, Mussaurus patagonicus Bonaparte y Vince, 1979, representado por individuos de pocos días de vida; los restos fueron hallados junto a cáscaras de huevos recuperadas pocos años antes en esa misma localidad. Un año después de este hallazgo, Casamiquela (1980) publica la presencia del género Plateosaurus en esa localidad, sobre la base de numeroso material postcraneano. Sin embargo, más tarde, esos materiales serían atribuidos a individuos adultos de Mussaurus patagonicus (Otero y Pol, 2013). En el año 2003 y más recientemente en 2012 y 2013, nuevas expediciones a la provincia de Santa Cruz lideradas por Diego Pol (Museo Paleontológico Egidio Feruglio, Trelew), resultaron en el hallazgo de nuevos y abundantes materiales de sauropodomorfos basales provenientes de la Formación Laguna Colorada. Los restos, que incluyen individuos juveniles, subadultos y adultos de Mussaurus, así como sus sitios de nidificación, permitieron el reconocimiento de una asociación extraordinaria (Smith et al., 2014).

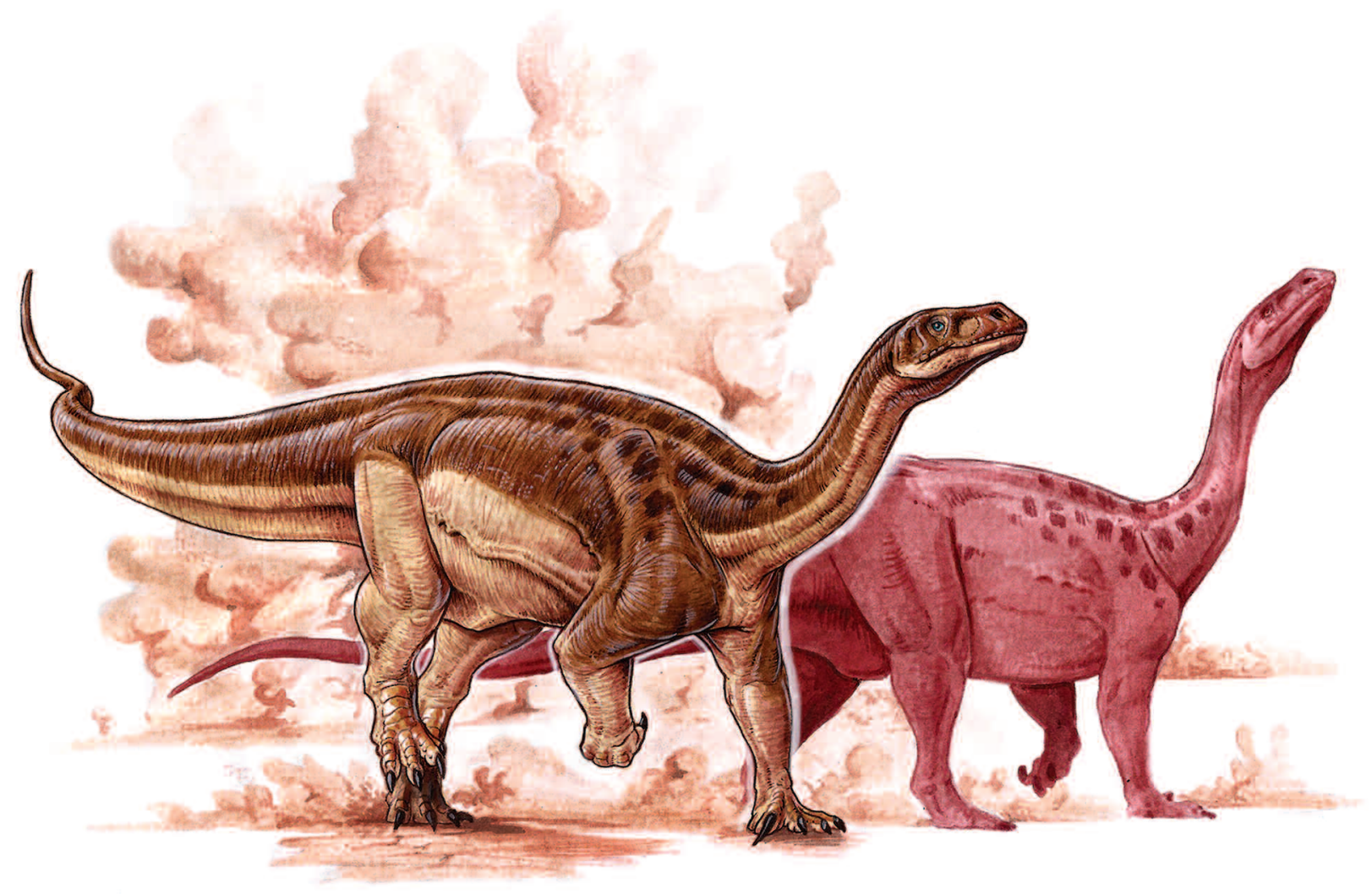

Figura 3. El sauropodomorfo basal Riojasaurus incertus de la Formación Los Colorados, provincia de La Rioja. Dibujo Gabriel Lio. 
Leonerasaurus taquetrensis Pol, Garrido y Cerda, 2011, de la Formación Las Leoneras (probablemente Toarciense, Jurásico Inferior) de la provincia de Chubut, es el segundo sauropodomorfo basal registrado en Patagonia. Leonerasaurus es un ejemplar incompleto del que se conoce parte de un dentario y restos postcraneanos, incluido un sacro con cuatro elementos vertebrales, condición que lo acerca al origen de Sauropoda (Pol et al., 2011).

\section{Sauropoda (Anexo 1)}

La definición de Sauropoda propuesta por Yates (2007a), basada en la rama usando a Melanorosaurus como taxón de referencia externo, ubica a Lessemsaurus sauropoides del Triásico Superior de la Formación Los Colorados, como un saurópodo junto al sudafricano Antetonitrus ingenipes Yates y Kitching, 2003. Más allá del hecho que ambos taxones presentan numerosos estados de caracteres compartidos con Sauropoda/Eusauropoda (Yates y Kitching, 2003; Pol y Powell, 2007; McPhee et al., 2014), la presencia de simplesiomorfías clave, particularmente en los miembros (e.g., morfología de la ulna y elementos de la mano y el ilion primitivo) exigen precaución al considerar a estos taxa como Sauropoda.

Amygdalodon patagonicus Cabrera, 1947 de la Formación Cerro Carnerero (Jurásico Medio) de la provincia del Chubut, es el saurópodo más primitivo hallado en Sudamérica, representado por materiales pertenecientes al menos a tres individuos. Amygdalodon fue tradicionalmente considerado como un eusaurópodo (Wilson, 2002; Rauhut, 2003; Upchurch et al., 2004) sobre la base de estados de caracteres derivados en las vértebras. Sin embargo, nueva información proveniente de la anatomía dentaria ubica a Amygdalodon por fuera de Eusauropoda (Carballido y Pol, 2010; Carballido y Sander, 2013).

Eusauropoda. Este clado incluye a todos los saurópodos más cercanamente relacionados a Saltasaurus que a Vulcanodon Raath, 1972 (Wilson y Sereno, 1998).

Los eusaurópodos basales de la Argentina están representados por Volkheimeria chubutensis Bonaparte, 1979 y por Patagosaurus fariasi Bonaparte, 1979 de la Formación Cañadón Asfalto (Jurásico Medio), provincia del Chubut. La primera especie está representada por un esqueleto incompleto, mientras que la segunda incluye varios especímenes
(Bonaparte, 1986; Rauhut, 2002). Sin embargo, estudios recientes basados en micromorfología dentaria corroboran que algunos individuos tradicionalmente referidos a Patagosaurus pertenecen a otro taxón (Holwerda et al., 2015). Neosauropoda. Neosauropoda fue definido por Salgado et al. (1997a) como el clado que incluye al ancestro común más reciente de Diplodocoidea y Camarasauromorpha y a todos sus descendientes.

Diplodocoidea. Neosaurópodos más cercanamente emparentados con Diplodocus Marsh, 1878 que con Saltasaurus (Wilson y Sereno, 1998).

El registro de saurópodos diplodocoideos de la Argentina incluye a los Dicraeosauridae, Rebbachisauridae y Diplodocidae. En este sentido, el primer registro de un diplodocoideo para América del Sur corresponde a un Dicraeosauridae hallado en 1991, grupo hasta ese entonces sólo conocido para el Jurásico Superior de Tanzania (Janensch, 1914, 1929). Es así que el hallazgo de Amargasaurus cazaui en la Formación La Amarga (Barremiense, Cretácico Inferior), provincia del Neuquén, fue de gran importancia considerando que, en aquel entonces, el registro de saurópodos para Sudamérica estaba sesgado a los titanosaurios. Amargasaurus está representado por un esqueleto casi completo, incluyendo parte del basicráneo y techo craneano, y por un espécimen referido, constituido por una vértebra cervical. Este dicreosáurido, con inusuales espinas neurales bífidas y extremadamente largas, junto a otros restos reportados también en la provincia del Neuquén (Coria et al., 2012, 2013a; Gallina et al., 2014a), constituyen posiblemente los últimos supervivientes de la familia Dicraeosauridae; los restantes miembros no sobrevivieron más allá del Jurásico Tardío (Whitlock, 2011).

Por más de quince años, Amargasaurus representó el único taxón de Dicraeosauridae para Sudamérica. Sin embargo, eso cambiaría en el año 2005 con el descubrimiento de Brachytrachelopan mesai Rauhut, Remes, Fechner, Cladera y Puerta, 2005, de la Formación Cañadón Calcáreo, provincia del Chubut. Brachytrachelopan no sólo incrementó el registro de esta familia en Sudamérica sino que también extendió su rango temporal hasta el Jurásico Superior (Titoniense). La característica más peculiar de este taxón, representado por un esqueleto parcial, es su cuello extremadamente corto, a lo que debe su nombre ("Dios de los pastores de cuello corto"). 
Sin lugar a dudas, los diplodocoideos mejor representados en la Argentina son los Rebbachisauridae. Este grupo fue conocido en nuestro país gracias al reporte de "Rebbachisaurus" tessonei Calvo y Salgado, 1995, proveniente de la Formación Candeleros (Cenomaniense inferior, Cretácico Superior), provincia del Neuquén. Para el momento en que " $R$ ". tessonei fue erigido, el único punto de comparación era Rebbachisaurus garasbae Lavocat, 1954, del Cretácico Superior de Marruecos (Lavocat, 1954), con quien compartía varias sinapomorfías. Sin embargo, con el pasar del tiempo, nuevos registros de rebaquisáuridos en diferentes partes del mundo sacaron a la luz el hecho de que muchos de los caracteres que diagnosticaban al género Rebbachisaurus eran propios de un grupo más inclusivo de diplodocoideos, los Rebbachisauridae. Frente a esto, Salgado et al. (2004) propusieron una nueva combinación para este taxón, Limaysaurus tessonei (Calvo y Salgado, 1995).

Apenas un año después de la descripción de Limaysaurus tessonei, otro rebaquisáurido, Rayososaurus agrioensis Bonaparte, 1996 fue dado a conocer a partir de materiales fragmentarios provenientes de la Formación Rayoso (Aptiense, Cretácico Inferior), provincia del Neuquén. Este único espécimen se halla compuesto por una escápula incompleta y porciones de fémur y fíbula. Bonaparte (1996) advirtió en él ciertas similitudes con Rebbachisaurus garasbae, en cuanto a la morfología de la escápula, por lo que propuso una nueva familia de saurópodos, los Rebbachisauridae (Bonaparte, 1997), el taxón hermano de Dicraeosauridae y Diplodocidae, con una distribución temporal puramente cretácica (Carballido et al., 2010; Whitlock, 2011). Actualmente se considera que Rayososaurus corresponde al Cretácico Superior, proveniente en realidad de los niveles inferiores de la formación cenomaniana Candeleros (Carballido et al., 2010).

Desde que Rayososaurus fuera dado a conocer, pasaron casi diez años sin nuevos registros de rebbachisáuridos para la Argentina. Cathartesaura anaerobica Gallina y Apesteguía, 2005 de la Formación Huincul (Cenomaniense superior, Cretácico Superior), provincia de Río Negro, rompió con casi una década de esterilidad en el registro de este grupo. Cathartesaura es conocido por un esqueleto fragmentario pobremente preservado, aunque la presencia de autapomorfías en las vértebras cervicales y caudales justifica su identidad taxonómica. Mención especial merece el especimen MMCHPv-49, proveniente también de la Formación Huincul de los alrededores de Villa el Chocón (provincia del Neuquén). Está compuesto por una serie vertebral cervicodorsal exquisitamente preservada, más elementos apendiculares y ha sido catalogado como Rebbachisauridae indet. (Haluza et al., 2012), aunque con algunas similitudes con su coetáneo de Río Negro.

Un año después de la nominación de Cathartesaura, se dan a conocer los restos de otro rebbachisáurido, colectado entre 1995 y 1996 por José Bonaparte. Se trata de Zapalasaurus bonapartei Salgado, Carvalho y Garrido, 2006 proveniente de la Formación La Amarga, provincia del Neuquén, que constituye el registro más antiguo de un rebbachisáurido para la Argentina, extendiéndose al Barremiense/Aptiense (Cretácico Inferior) (Fig. 4).

Comahuesaurus windhauseni Carballido, Salgado, Pol, Canudo y Garrido, 2012 de la Formación Lohan Cura (Aptiense/Albiense, Cretácico Inferior), también de la provincia del Neuquén junto a Zapalasaurus y Amazonsaurus, forma parte de la primera radiación de este grupo para nuestro subcontinente.

Por último, el rebbachisáurido patagónico Katepensaurus goicoecheai Ibiricu, Casal, Martínez, Lamanna, Luna y Salgado, 2013 de la Formación Bajo Barreal (Cenomaniense/Turoniense, Cretácico Superior), provincia del Chubut, es conocido a partir de vértebras cervicales, dorsales y caudales. Ibiricu et al. (2013) consideran a Katepensaurus un posible miembro de los Limaysaurinae, dentro de los Rebbachisauridae.

Finalmente, el hallazgo más reciente de un diplodocoideo para la Argentina corresponde a Leinkupal laticauda GaIlina, Apesteguía, Canale y Haluza, 2014b, de la Formación Bajada Colorada (Berriasiense/Valanginiense, Cretácico Inferior), provincia del Neuquén. Si bien el material es fragmentario y mal preservado, se identifican caracteres que permiten asignarlo a la familia Diplodocidae, constituyendo el registro más moderno para este grupo y el primero para Sudamérica (Gallina et al., 2014b).

Camarasauromorpha. El clado Camarasauromorpha fue propuesto por Salgado et al. (1997a, p. 9) para incluir al ancestro común de Camarasauridae y Titanosauriformes, más todos sus descendientes. Este clado representa, junto a 


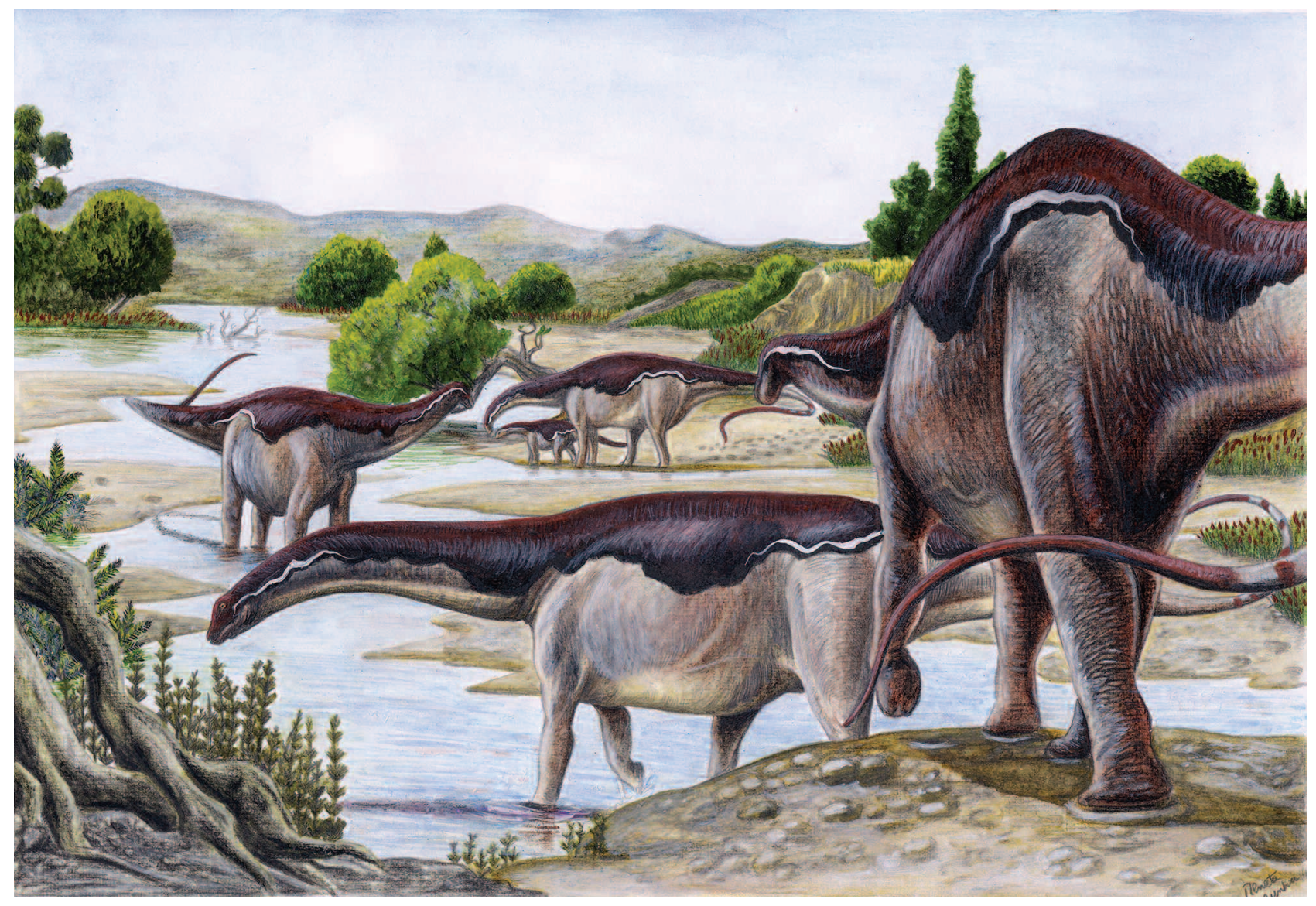

Figura 4. El diplodocoideo rebachisáurido Zapalasaurus bonapartei de la Formación La Amarga, provincia del Neuquén. Dibujo de Renata Cunha.

Macronaria (Wilson y Sereno, 1998), la primera radiación de los Neosauropoda no diplodocoideos, la cual está escasamente representada en Sudamérica (Salgado et al., 1997a).

Tehuelchesaurus benitezi Vickers-Rich, Rich-Thomas, Giménez, Cúneo, Puerta y Vacca, 1999 de la Formación Cañadón Calcáreo (Kimmeridgiense/Titoniense, Jurásico Superior), provincia del Chubut, representa uno de los registros de Neosauropoda más antiguos para Sudamérica. Si bien originalmente fue relacionado con los "Cetiosauridae" (Rich et al., 1999), la presencia de vértebras dorsales posteriores opistocélicas ubican a Tehuelchesaurus entre los Camarasauromorpha (Carballido et al., 2011a).

Titanosauriformes. Este clado de neosaurópodos fue erigido y definido por Salgado et al. (1997a, p. 12) como el ancestro común más reciente de Giraffatitan brancai (Janensch, 1914), Chubutisaurus insignis del Corro, 1975 y Titanosauria, más todos sus descendientes. Los titanosauriformes y todos sus clados anidados, se caracterizan mayormente por atributos postcraneanos, particularmente apendiculares, entre los que se destacan un proceso preacetabular del ilion semicircular y el fémur con el tercio proximal medialmente inclinado (Wilson y Sereno, 1998; Wilson y Carrano, 1999).

El primer titanosauriforme basal reportado en la Argentina es Chubutisaurus insignis, de la Formación Cerro Barcino (Cenomaniense, Cretácico Superior), provincia del Chubut. Chubutisaurus fue encontrado en 1961 y colectado por del Corro en 1965. Originalmente ubicado dentro de una familia propia (Chubutisauridae; del Corro, 1975), Chubutisaurus fue posteriormente catalogado como un Sauropoda incertae sedis debido a la ausencia de caracteres compartidos con otros grupos (Salgado, 1993). Un exhaustivo estudio de la anatomía de Chubutisaurus, así como su contextualización en un marco filogenético actualizado, ubican a este taxón dentro de las formas basales de titanosauriformes (CarbaIlido et al., 2011b) (Fig. 5).

En la Formación Lohan Cura (Aptiense, Cretácico Inferior), 
provincia del Neuquén, se han registrado otros dos titanosaurifomes basales: Agustinia ligabuei Bonaparte, 1999 y Ligabuesaurus leanzai Bonaparte, González Riga y Apesteguía 2006. Agustinia ligabuei, representado por un único espécimen, fue originalmente referido a una familia propia, Agustiniidae, con afinidades con los Rebbachisauridae, sobre la base de la morfología general de las espinas neurales y la arquitectura laminar (Bonaparte, 1999). La presencia de osteodermos, sin embargo, relaciona a este taxón con Titanosauria (Upchurch et al., 2004). Ligabuesaurus está representado por un esqueleto incompleto y fue originalmente referido como un titanosaurio basal por Bonaparte et al. (2006), quienes subrayaron la presencia de miembros an- teriores relativamente largos, sugiriendo que esta condición no era exclusiva de los Brachiosauridae. Estudios filogenéticos recientes ubican a este taxón como un miembro basal de Titanosauriformes (Carballido y Sander, 2013) o bien como un Somphospondyli basal (D'Emic, 2012).

Finalmente, Amargatitanis macni Apesteguía, 2007 de la Formación La Amarga (Barremiense, Cretácico Inferior) de la provincia del Neuquén, fue referido inicialmente a Titanosauria, sobre la base de restos fragmentarios de vértebras caudales, escápula, fémur y astrágalo (Apesteguía, 2007). Sin embargo, estudios recientes han puesto en duda sus afinidades con los titanosaurios, principalmente por su preservación fragmentaria y la falta de sinapomorfías del clado

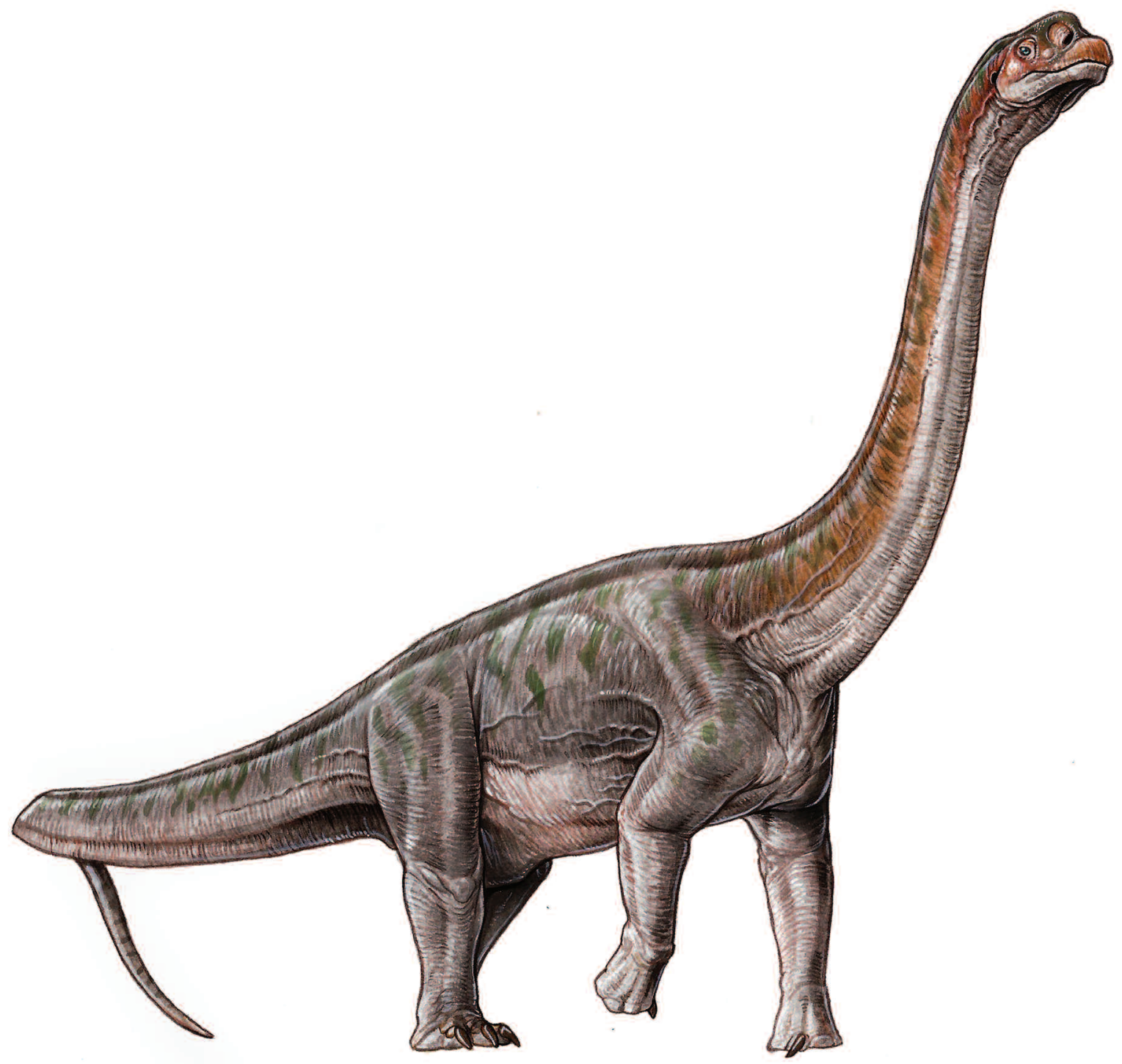

Figura 5. El titanosauriforme basal Chubutisaurus insignis de la Formación Cerro Barcino, provincia del Chubut. Dibujo Gabriel Lio. 
Titanosauria (D’Emic, 2012; Mannion et al., 2013).

Titanosauria. Sin lugar a dudas los titanosaurios son los saurópodos mejor representados en el registro fósil del territorio argentino, con aproximadamente 30 especies válidas. Titanosauria se define como el clado que incluye al ancestro común más reciente de Andesaurus delgadoi Calvo y Bonaparte, 1991 y Saltasaurus, más todos sus descendientes (Wilson y Upchurch, 2003).

El conocimiento de los saurópodos en la Argentina vino de la mano de los titanosaurios y del paleontólogo británico Richard Lydekker, quien viajó a nuestro país en 1893 y 1894 para realizar descripciones exhaustivas de numerosos fósiles de vertebrados colectados por expediciones previas del Museo de La Plata. En sus Contributions to the Study of the Fossil Vertebrates of Argentina (Contribuciones al estudio de los fósiles de vertebrados de la Argentina), Lydekker (1893) reconoció la presencia del género Titanosaurus, erigido por él mismo casi diez años antes sobre la base de materiales colectados en la India. Asimismo, nominó varias especies de titanosaurios (ver más adelante), erigiendo además a la familia homónima.

Entre 1921 y 1924 el Museo de La Plata llevó a cabo una serie de exploraciones en el Norte de la Patagonia, lideradas por Santiago Roth y Walter Schiller, resultando en varios titanosaurios colectados, los que fueron estudiados en La Plata por Friedrich von Huene, profesor alemán de la Universidad de Tübingen, Alemania.

No fue sino hasta la década del ochenta que los registros de este clado de macronarios se hicieron recurrentes gracias a las expediciones sistemáticas realizadas por el paleontólogo José F. Bonaparte y que luego continuarán sus discípulos. Se presenta a continuación el registro de titanosaurios bajo un orden estratigráfico.

El registro de este clado en la Argentina comienza en el Cretácico Superior bajo. Andesaurus delgadoi de la Formación Candeleros (Cenomaniense inferior), provincia del Neuquén, corresponde no sólo a uno de los registros más antiguos de titanosaurios, sino también a la forma más primitiva de este grupo (como ya vimos, Andesaurus forma parte de la definición filogenética del clado), debido principalmente a la ausencia de procelia en las vértebras caudales medias, carácter que diagnostica a Lithostrotia (Upchurch et al., 2004). La identidad de Andesaurus como un titanosaurio está dada, entre otros caracteres, por la presencia de pleurocelos en vértebras dorsales ubicados en una depresión ovoide y vértebras caudales anteriores procélicas (Mannion y Calvo, 2010).

Argentinosaurus huinculensis Bonaparte y Coria, 1993 de la Formación Huincul (Cenomaniense superior, Cretácico Superior), provincia del Neuquén, es problemático debido a que diferentes opiniones han sido puestas en juego en cuanto a la interpretación de su anatomía. Salgado et al. (1997a) indican que las articulaciones extra hipósfeno-hipantro en las vértebras dorsales posteriores están ausentes, por lo que consideran a este dinosaurio relacionado a los titanosaurios más derivados. Wilson (2002) lo considera un macronario basal debido a la presencia de vértebras dorsales posteriores opistocélicas. No debe descartarse ninguna posición hasta tanto los materiales sean reinterpretados.

Las capas cenomanienses de las provincias del Chubut y Santa Cruz han contribuido prolíficamente al conocimiento de los titanosaurios al sur del río Chubut, todos ellos provenientes de las formaciones Bajo Barreal (Cenomaniense/ Turoniense) y Lago Colhue Huapi (Coniaciense/Maastrichtiense). Argyrosaurus superbus Lydekker, 1893, hallado en Chubut en niveles actualmente atribuibles a la Formación Lago Colhue Huapi, es uno de los primeros titanosaurios dados a conocer en la Argentina (Lydekker, 1893). En su descripción original, Lydekker (op. cit.) implícitamente nomina como holotipo un miembro anterior casi completo, hoy en día restaurado y exhibido en el Museo de La Plata. Muchos otros materiales han sido referidos a este taxón por Lydekker y autores posteriores (e.g., Powell, 2003), sobre la base de similitudes superficiales y proporciones, pero sin apomorfías compartidas. Algunos de esos materiales (los especímenes PVL 4628 y MACN-CH 217, colectados por José Bonaparte en la misma unidad) constituyen un taxón diferente, Elaltitan lilloi Mannion y Otero, 2012, representado por un esqueleto parcial que incluye elementos vertebrales dorsales y caudales, así como huesos de los miembros.

El titanosaurio Epachthosaurus sciuttoi Powell, 1990 proviene de la Formación Bajo Barreal; el holotipo es una vértebra dorsal y el paratipo una réplica de un esqueleto incompleto en su matriz original y expuesto del lado lateral. Cuenta además con un espécimen referido que representa 
uno de los titanosaurios más completos conocidos hasta el momento (Martínez et al., 2004).

Aeolosaurus colhuehuapensis Casal, Martínez, Luna, Sciutto y Lamanna, 2007 fue hallado en el lago Colhue Huapi, provincia del Chubut (Formación Lago Colhue Huapi, Casal et al., 2015). Se distingue de $A$. rionegrinus de la Cuenca Neuquina (ver más abajo) por la presencia de profundas fosas en las vértebras caudales anteriores, una delgada lámina que une la base de la lámina prespinal y el borde inferior de la faceta articular en la parte medial de las prezigapófisis (que define una fosa a ambos lados de la lámina prespinal) y vértebras caudales con el cono articular posterior en posición media (Casal et al., 2007, p. 55).

Drusilasaura deseadensis Navarrete, Casal y Martínez, 2011, proviene de la Formación Bajo Barreal, en la provincia de Santa Cruz y cuenta con un esqueleto bastante incompleto, compuesto de vértebras dorsales y caudales, una vértebra sacra y una escápula. Se aguarda la inclusión de este taxón en una matriz filogenética para establecer sus relaciones de parentesco.

Un gran número de titanosaurios han sido registrados en capas turonienses/coniacienses, todos ellos provenientes de la zona norte de la Cuenca Neuquina y la mayoría reportados en la zona de influencia de Rincón de los Sauces y el sur de Mendoza. Rinconsaurus caudamirus Calvo y González Riga, 2003, de la provincia del Neuquén y Mendozasaurus neguyelap González Riga, 2003, de la provincia que le da origen a su nombre, son dos titanosaurios que provienen de capas provisionalmente referidas a la Formación o Subgrupo Río Neuquén (Cretácico Superior) (González Riga, 2003; Calvo y González Riga, 2003). Esto significa que, bajo el actual esquema estratigráfico propuesto por Garrido (2010), correspondería a la Formación Portezuelo o a la Formación Los Bastos. Tanto Rinconsaurus como Mendozasaurus están originalmente representados por una serie de vértebras caudales, que corresponden al holotipo, más un gran número de especímenes que corresponden a los paratipos. Rinconsaurus se caracteriza, entre otros caracteres, por la presencia de una combinación de vértebras caudales anficélicas, biconvexas y opistocélicas. El análisis filogenético original ubica a Rinconsaurus anidado junto a Aeolosaurus a través de "prezigapófisis caudales relativamente largas" (Calvo y González Riga, 2003). Sin embargo, Rinconsaurus no posee tres de las sinapomorfías que diagnostican a Aeolosaurini (sensu Franco-Rosas et al., 2004): cuerpos vertebrales caudales anteriores y medios con el margen anterior inclinado hacia delante; arcos neurales dispuestos sobre el borde anterior del centro en las vértebras caudales medias; espina neural inclinada hacia adelante, al menos en las caudales medias.

Dos años después de su descripción original, fueron descubiertos nuevos restos asignables a Mendozasaurus, correspondientes a vértebras cervicales, lo que posiblitó expandir su diagnosis original (González Riga, 2005). Asimismo, la presencia de elementos cervicales permitió, posteriormente incluirlo en el clado Lognkosauria, junto al gigantesco Futalognkosaurus dukei Calvo, Porfiri, González Riga y Kellner, 2007a de la Formación Portezuelo (Turoniense/Coniaciense, Cretácico Superior), de la provincia del Neuquén. Si bien Lognkosauria es definido como el ancestro común más reciente de Mendozasaurus neguyelap y Futalognkosaurus dukei y todos sus descendientes, y su diagnosis se basa principalmente en caracteres cervicales (Calvo et al., 2007a), las probables sinapomorfías propuestas para este clado están más ampliamente distribuidas, como lo demuestran recientes análisis filogenéticos (Gallina y Apesteguía, 2011; Salgado et al., 2014; Gallina y Otero, 2015).

La rica Formación Portezuelo proporcionó otros dos taxones, también provenientes del norte de la Cuenca Neuquina: Malarguesaurus florenciae González Riga, Previtera, y Pirrone, 2009 de la provincia de Mendoza y Muyelensaurus pecheni Calvo, González Riga y Pirrone, 2007 de la provincia del Neuquén. Malarguesaurus está representado por un individuo compuesto por una serie vertebral caudal incompleta y restos apendiculares muy fragmentarios. El análisis filogenético original ubica a este taxón como un Somphospondyli basal (González Riga et al., 2009), junto a Phuwiangosaurus Martin, Buffetaut, Suteethorn, 1994, resultado compartido por Mannion et al. (2013), mientras que Carballido y Sander (2013) lo sitúan como un Titanosauria basal. En ambos análisis Malarguesaurus fue podado del consenso debido a su registro fragmentario. A pesar de ello, es probable que este taxón se encuentre filogenéticamente por fuera de Titanosauria debido a la ausencia de procelia 
marcada tanto en las vértebras caudales anteriores como medias.

Muyelensaurus pecheni, hallado en las cercanías de la localidad neuquina de Rincón de Los Sauces, está representado por al menos cinco individuos, en una asociación monoespecífica que incluye material craneano y un postcráneo muy completo (Calvo et al., 2007b).

De sedimentos santonienses (Cretácico Superior) proviene Petrobrasaurus puestohernandezi Filippi, Canudo, Salgado, Garrido, García, Cerda y Otero, 2011 de la Formación Plottier, provincia del Neuquén, compuesto por un individuo cuya preservación incluye un fragmento de vértebra cervical, vértebras dorsales y caudales y elementos apendiculares. Filippi et al. (2011a) indican que Petrobrasaurus se ubica filogenéticamente en una politomía en la base de Titanosauria (aunque no incluyen un cladograma con la posición de este taxón), con lo cual lo consideran un Titanosauria incertae sedis. Considerando la presencia de vértebras caudales anteriores y medias opistocélicas, muy probablemente se incluya en Lithostrotia.

Bonitasaura salgadoi Apesteguía, 2004 es otro taxón santoniense (Formación Bajo de La Carpa) de la provincia de Río Negro, representado por un único espécimen, muy completo y exquisitamente preservado. Bonitasaura es, sin dudas, uno de los titanosaurios mejor conocidos, del que se han realizado exhaustivas descripciones, incluyendo un análisis paleohistológico y tafonómico (Apesteguía, 2004; Pérez et al., 2009; Gallina, 2011, 2012; Gallina y Apesteguía, 2011, 2015). Una de las características más notables de este taxón es la presencia del margen posterior del dentario edéntulo y afilado, con evidencias de alta vascularización, lo que permitió a Apesteguía (2004) inferir la presencia de un "pico", o una estructura similar. La presencia de centros vertebrales no fusionados al arco neural, sumado al abundante tejido fibrolamelar y a la ausencia de un Sistema Fundamental Externo, sugieren que el animal representado por el material holotipo de Bonitasaura salgadoi continuaba su crecimiento al momento de morir (Apesteguía, 2004; GaIlina, 2011, 2012). Originalmente, este taxón fue relacionado a Nemegtosaurus Nowinski, 1971, Quaesitosaurus Kurzanov y Bannikov, 1983 y Rapetosaurus Curry Rogers y Forster, 2001, sobre la base de atributos craneanos compartidos, aunque sin la inclusión de un análisis filogenético (Apeste- guía, 2004). Análisis filogenéticos recientes han demostrado la relación de Bonitasaura con formas sudamericanas como el clado Lognkosauria, Muyelensaurus y Rinconsaurus (Gallina y Apesteguía, 2011; Salgado et al., 2014; Gallina y Otero, 2015).

La Formación Anacleto (Campaniense, Cretácico Superior), aflorante en las provincias de Río Negro y Neuquén es una de las unidades con el registro más rico de titanosaurios. Neuquensaurus australis (Lydekker, 1893) y Laplatasaurus araukanicus Huene, 1929 corresponden a los primeros dinosaurios reportados para esta unidad. Neuquensaurus (= Titanosaurus) australis fue nominado originalmente por Lydekker (1893) sobre la base de seis vértebras caudales, las cuales corresponden al holotipo, y posteriormente ampliado incluyendo el sacro (D'Emic y Wilson, 2011). Más tarde, Huene (1929) reconoce la existencia de dos "morfotipos" entre todos los materiales originalmente referidos a este taxón: uno más grácil, al que denominó Titanosaurus australis, y otro más robusto al que llamó Titanosaurus robustus, aunque sin proporcionar una diagnosis, lo cual lo condenó posteriormente al status de nomen dubium (Powell, 2003; Wilson y Upchurch, 2003). Salgado et al. (2005) describen nuevos materiales correspondientes a dos especímenes asignables a Neuquensaurus australis, provenientes de la localidad de Cinco Saltos, Río Negro, ampliando la diagnosis para esta especie. Posteriormente, un análisis osteológico del esqueleto apendicular del género arrojó luz sobre la identidad de Neuquensaurus robustus, presente tanto en los materiales de Lydekker (1893), Huene (1929) y Salgado et al. (2005), quedando pendiente la revisión del esqueleto axial para corroborar definitivamente la identidad taxonómica de $N$. robustus (Otero, 2010) (Fig. 6).

Los materiales originalmente referidos a Laplatasaurus araukanicus Huene, 1929 provienen de cinco localidades diferentes del Norte de Patagonia e incluyen restos postcraneanos de múltiples especímenes y diferentes condiciones de preservación. Esto, sumado a la ausencia de mapas de los sitios de hallazgos y de una diagnosis formal, hizo de $L a$ platasaurus un taxón problemático. Bonaparte y Gasparini (1979) hicieron el primer esfuerzo por aclarar el panorama en cuanto a la identidad taxonómica de este taxón, revisando los materiales y designando un lectotipo (una tibia y una fíbula). Pese a esto, la validez de los restantes materia- 


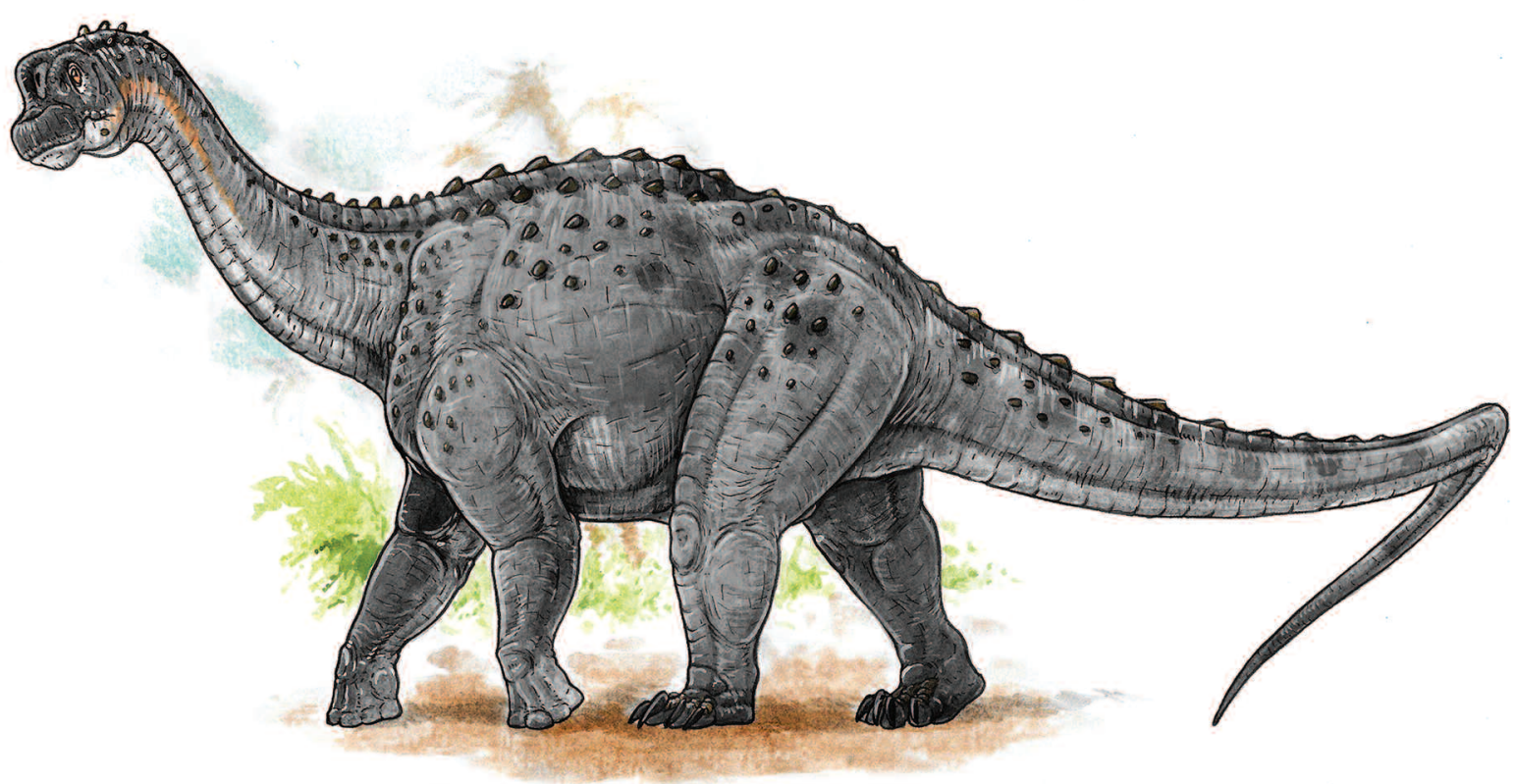

Figura 6. El titanosaurio saltasaurino Neuquensaurus australis de la Formación Anacleto, provincia del Neuquén. Dibujo Gabriel Lio.

les asignados al género permaneció sin resolver durante aproximadamente 25 años. Recientemente, Gallina y Otero (2015) revisaron todos los materiales referidos a Laplatasaurus, discutieron su validez y redescribieron el lectotipo, incluyéndolo en una matriz filogenética por vez primera. El nombre de la especie fue retenido sólo para el lectotipo, en congruencia con Wilson y Upchurch (2003), mientras que el resto de los materiales referidos, sea por ausencia de elementos solapados o carencia de caracteres compartidos, fue designado como Lithostrotia indet., excepto los materiales de Rancho de Ávila, los cuales han sido referidos a cf. Bonitasaura por Gallina y Otero (2015).

Pellegrinisaurus powelli Salgado, 1996 proveniente de las cercanías de Cinco Saltos y Lago Pellegrini, provincia de Río Negro se compone de vértebras dorsales, una secuencia de 26 vértebras caudales y una porción proximal de fémur.

Reportado en Sierra Barrosa, Barrosasaurus casamiquelai Salgado y Coria, 2009 se compone de tres vértebras dorsales y se diferencia de otros titanosaurios coetáneos (e.g., Neuquensaurus, Laplatasaurus, Pellegrinisaurus) por características únicas en las láminas del arco neural (Salgado y Coria, 2009).
La localidad neuquina de Rincón de los Sauces ha contribuido con un número significativo de titanosaurios campanienses, a través del Museo Municipal "Argentino Urquiza". Pitekunsaurus macayai Filippi y Garrido, 2008 es un interesante titanosaurio, cuyo material cuenta con un basicráneo y restos de postcráneo. Si bien los autores proponen cierta afinidad con Rinconsaurus sobre la base de la morfología vertebral caudal, su inclusión en una matriz filogenética por Coria et al. (2013b) lo relaciona con Aeolosaurini. Narambuenatitan palomoi Filippi, García y Garrido, 2011 es otro titanosaurio de los alrededores de Rincón de los Sauces que incluye material craneano, además de elementos vertebrales y apendiculares. Narambuentatitan se ubica filogenéticamente como un Lithostrotia no eutitanosaurio, muy relacionado a Epachthosaurus, compartiendo con este último la presencia de vértebras caudales medias con una espina neural laminar y anteroposteriormente elongada (Filippi et al., 2011b).

Finalmente, Overosaurus paradasorum Coria, Filippi, Chiappe, García, y Arcucci, 2013b es un titanosaurio de tamaño reducido, representado por una serie vertebral axial casi completa y muy bien preservada, a la que le falta sólo 
la parte más anterior del cuello y la más posterior de la cola. Desafortunadamente, del esqueleto apendicular sólo se han preservado los iliones. El análisis filogenético presentado por Coria et al. (2013b) muestra a Overosaurus como un AeoIosaurini. Overosaurus representa una oportunidad única para el estudio de cambios seriales axiales en titanosaurios.

Dreadgnouthus schrani Lacovara, Lamanna, Ibiricu, Poole, Schroeter, Ullmann, Voegele, Boles, Carter, Fowler, Egerton, Moyer, Coughenour, Schein, Harris, Martínez y Novas, 2014 proveniente de la Formación Cerro Fortaleza, es un titanosaurio no Lithostrotia de gran porte que incluye un $65 \%$ del esqueleto, lo que lo convierte en uno de los titanosaurios de talla gigantesca mejor representados. Otro titanosaurio de gran tamaño fue hallado en la provincia de Santa Cruz, Puertasaurus reuilli Novas, Salgado, Calvo, Agnolín, 2005 de la Formación Pari Aike (Maastrichtiense, Cretácico Superior). Al igual que Argentinosaurus, Puertasaurus posee vértebras dorsales extremadamente grandes y robustas, aunque sin material apendicular, por lo que estimaciones de masa corporal son difíciles de abordar sin un alto componente especulativo. Respecto de la proveniencia de Dreadgnothus y Puertasaurus, es necesario destacar que estudios recientes sugieren que la zona de hallazgo de estos taxones corresponde a la Formación Mata Amarilla, cuya reciente datación radimétrica ha arrojado una edad cenomaniense para esta formación (Varela et al., 2012a,b), poniendo en duda la hipótesis de la presencia de gigantismo extremo hacia finales del Cretácico. Finalmente, los registros de titanosaurios en sedimentos campanienses/maastrichtienses están representados en las provincias de Río Negro y Salta.

Aeolosaurus rionegrinus Powell, 1990, procedente originalmente de la Formación Angostura Colorada (Campaniense-Maastrichtiense), provincia de Río Negro, está compuesto por vértebras caudales y parte del esqueleto apendicular. Salgado et al. (1997b) reportan la presencia de este género en la Formación Los Alamitos (CampanienseMaastrichtiense), en la misma provincia, sobre la base de elementos caudales y apendiculares. Aeolosaurus le da nombre a la subfamilia Aeolosaurini (Franco-Rosas et al., 2004), cuya diagnosis está basada en caracteres caudales.

Antarctosaurus wichmannianus Huene, 1929, de la Formación Allen (Maastrichtiense), proveniente de la provincia de Río Negro fue uno de los primeros saurópodos de la Ar- gentina en ser nominados. Su holotipo consta de un cráneo incompleto y un postcráneo incompleto, mayormente constituido por elementos apendiculares. Al día de hoy se espera una revisión de esta especie que incluya una descripción actualizada del material craneano.

Rocasaurus muniozi Salgado y Azpilicueta, 2000 es un Saltasaurini proveniente de la Formación Allen, cerca de la localidad rionegrina de General Roca. Su holotipo está compuesto por un individuo juvenil y hay varios ejemplares referidos.

También de la provincia de Río Negro y de la Formación Allen, Bonatitan reigi Martinelli y Forasiepi, 2004 es un titanosaurio que consta de un basicráneo, más una serie de materiales postcraneanos originalmente incluidos en el holotipo, más un ejemplar referido (Martinelli y Forasiepi, 2004). Estos autores consideraron a este taxón como un miembro del clado Saltasaurinae (sensu Powell, 1992), aunque sin incluirlo en una matriz filogenética. Recientemente, Salgado et al. (2014) redescribieron los materiales de este género, incluyendo elementos óseos inéditos y concluyeron sobre la existencia de cinco ejemplares. El análisis filogenético presentado por Salgado et al. (2014) muestra a Bonatitan como un miembro basal de un clado más inclusivo que abarca a Aeolosaurini y Saltasauridae.

Panamericansaurus schroederi Calvo y Porfiri, 2010, proveniente de la Formación Allen en la provincia del Neuquén, es un Aeolosaurini diagnosticado por atributos presentes en las vértebras caudales y en el húmero.

En 1980 se reporta la presencia de un titanosaurio en la provincia de Salta, Saltasaurus loricatus, proveniente de la Formación Lecho (Maastrichtiense). Este titanosaurio, miembro del clado Saltasaurini (sensu Salgado y Bonaparte, 2007), está representado por varios individuos, incluyendo material craneano y una inusual coraza de osteodermos, lo que le valió el nombre específico. Powell (1986, 2003) ofrece una descripción de estos materiales, incluyéndolos en una matriz filogenética y relacionándolos con Neuquensaurus australis. Cerda y Powell (2010), por su parte, aportaron al conocimiento detallado de la microestructura de las placas dérmicas de Saltasaurus, reconociendo un origen casi exclusivamente por mineralización directa. Recientemente, Zurriaguz y Powell (2015) dieron nueva luz al conocimiento de la anatomía vertebral presacra de este titanosaurio, incluyendo una exhaustiva y actualizada descripción tanto de 
las láminas como de las fosas vertebrales, demostrando que estas últimas estructuras poseen un valor sistemático mucho mayor de lo que se pensaba previamente.

\section{PERSPECTIVA GENERAL}

El registro argentino de sauropodomorfos es, en líneas generales, variado, completo y virtualmente continuo, abarcando formas antiguas y primitivas desde el Triásico Superior, a formas modernas y derivadas, en el Cretácico Superior. Sin lugar a dudas este último período es el que ha aportado la mayor cantidad de taxones, correspondientes todos ellos a saurópodos titanosaurios. Sin embargo, esta situación ha comenzado a nivelarse en los últimos años.

El registro de sauropodomorfos en el intervalo Triásico Superior-Jurásico Inferior es un claro ejemplo de ello. En los últimos diez años las formas basales han pasado a tener un papel protagónico en la Argentina debido, por un lado, a los descubrimientos tanto de formas triásicas, como Panphagia (Martínez y Alcober, 2009; Martínez et al., 2012) y Chromogisaurus (Ezcurra, 2010; Martínez et al., 2012), así como de formas jurásicas, como Adeopapposaurus (Martínez, 2009), Leyesaurus (Apaldetti et al., 2011) y Leonerasaurus (Pol et al., 2011). Asimismo, nuevos aportes al conocimiento de taxones ya conocidos han arrojado luz sobre aspectos anatómicos previamente no considerados, así como nuevas interpretaciones sobre sus relaciones filogenéticas, como es el caso de Lessemsaurus (Pol y Powell, 2007), Eoraptor (Sereno et al., 2012) y Coloradisaurus (Apaldetti et al., 2012, 2014). Por su parte, Mussaurus patagonicus fue conocido durante más de veinte años a partir de restos de pichones reportados por Bonaparte y Vince (1979). Esos ejemplares (con claros atributos plesiomórficos) fueron incluidos en análisis filogenéticos recuperando una posición basal dentro de Sauropodomorpha (Upchurch et al., 2007). Esta situación se revirtió al conocerse que los materiales previamente asignados por Casamiquela (1980) a Plateosaurus, en realidad correspondían a ejemplares adultos de Mussaurus (Otero y Pol, 2013) y que las relaciones de parentesco de este último lo acercan a Sauropoda.

El registro de saurópodos correspondiente a la transición Jurásico Superior/Cretácico Inferior ha sido tradicionalmente un espacio semivacío que ha comenzado a llenarse con los registros de Tehuelchesaurus Vickers-Rich, Rich-
Thomas, Giménez, Cúneo, Puerta y Vacca, 1999 y Brachytrachelopan, del Jurásico Superior y un creciente número de formas del Cretácico Inferior, como Comahuesaurus, Zapalasaurus y Leinkupal (Diplodocoidea) y Ligabuesaurus, Agustinia y Amargatitanis (Titanosauriformes), que dan cuenta de una insospechada diversidad de clados de saurópodos para ese momento del Mesozoico.

Más allá de todos estos taxones que se han dado a conocer en los últimos años, intensivos trabajos de campo están siendo realizados actualmente por distintos museos y centros de investigación, a lo largo y ancho de nuestro país, a partir de los cuales se está colectando una prometedora masa crítica de materiales. Es de esperar que, una vez finalizada la preparación de esos nuevos ejemplares, pueda tenerse un panorama más acabado de la variada fauna de sauropodomorfos del Mesozoico de la Argentina.

\section{AGRADECIMIENTOS}

A G. Casal (UNPSJB) por el aporte de bibliografía y datos inéditos sobre la procedencia de materiales del sur de Chubut. Un agradecimiento especial a G. Lio y a las siguientes personas e instituciones, que permitieron gentilmente la reproducción de las siguientes figuras: F. Novas (Figura 3), Museo Paleontológico Egidio Feruglio (Figura 5), Museo "Ernesto Bachmann" (Figura 6). P. Gallina y R. Martínez aportaron valiosos comentarios que han enriquecido este trabajo. A Zulma Gasparini, por todo el apoyo brindado y sobre todo por su amistad.

\section{REFERENCIAS}

Apaldetti, C., Pol, D. y Yates, A. 2012. The postcranial anatomy of Coloradisaurus brevis (Dinosauria: Sauropodomorpha) from the Late Triassic of Argentina and its phylogenetic implications. Palaeontology 56: 277-301.

Apaldetti, C., Martínez, R.N., Alcober, O.A. y Pol, D. 2011. A new basal sauropodomorph (Dinosauria: Saurischia) from Quebrada del Barro Formation (Marayes-El Carrizal Basin), Northwestern Argentina. PLoS ONE 6: e26964. Doi:10.1371/ journal.pone.0026964.

Apaldetti, C., Martínez, R.N., Pol, D. y Souter, T. 2014. Redescription of the skull of Coloradisaurus brevis (Dinosauria, Sauropodomorpha) from the Late Triassic Los Colorados Formation of the Ischigualasto-Villa Unión Basin, northwestern Argentina. Journal of Vertebrate Paleontology 34: 1113-1132.

Apesteguía, S. 2004. Bonitasaura salgadoigen. et sp. nov.: a beaked sauropod from the Late Cretaceous of Patagonia. Naturwissenschaften 91: 493-497.

Apesteguía, S. 2007. The sauropod diversity of the La Amarga Formation (Barremian), Neuquén (Argentina). Gondwana Research 12: $533-546$.

Bonaparte, J.F. 1967. Dos nuevas "faunas" de reptiles triásicos de Argentina. I International Symposium of Gondwana (Mar del Plata), Actas 1: 286-306.

Bonaparte, J.F. 1971. Los tetrápodos del sector superior de la Formación Los Colorados, La Rioja, Argentina. Opera Lilloana 23: 1-183. 
Bonaparte, J.F. 1978. Coloradia brevis n. g. et n. sp. (Saurischia Prosauropoda), dinosaurio Plateosauridae de la Formación Los Colorados, Triásico superior de La Rioja, Argentina. Ameghiniana 15: 327-332.

Bonaparte, J.F. 1986. Les Dinosaures (Carnosaures, Allosaurides, Sauropodes, Cetiosaurides) du Jurassique Moyen de Cerro Cóndor (Chubut, Argentina). Annales de Paleontologie 72: 325-386.

Bonaparte, J.F. 1996. Cretaceous tetrapods of Argentina. En: G. Arratia (Ed.), Contributions of Southern South America to Vertebrate Paleontology, Münchner Geowissenchaftliche Abhandlngen, München (A) 30: 73-130.

Bonaparte, J.F. 1997. Rayososaurus agrioensis Bonaparte, 1995. Ameghiniana, Suplemento Resúmenes 34: 116R.

Bonaparte, J.F. 1999. An armoured sauropod from the Aptian of Northern Patagonia, Argentina. En: Y. Tomida, T.H. Rich y P. Vickers-Rich (Eds.), Proceedings of the Second Gondwanan Dinosaur Symposium. National Science Museum Monographs, Tokyo, p. 1-12.

Bonaparte, J.F. y Gasparini, Z.B. 1979. Los saurópodos de los grupos Neuquén y Chubut, y sus relaciones cronológicas. $5^{\circ}$ Congreso Geológico Argentino (Neuquén), Actas 2: 39-406.

Bonaparte, J.F. y Vince, M. 1979. El hallazgo del primer nido de dinosaurios triásicos (Saurischia, Prosauropoda), Triásico superior de Patagonia, Argentina. Ameghiniana 76: 173-782.

Bonaparte, J.F., González Riga, B.J. y Apesteguía, S. 2006. Ligabuesaurus leanzai gen. et sp. nov. (Dinosauria, Sauropoda), a new titanosaur from the Lohan Cura Formation (Aptian, Lower Cretaceous) of Neuquén, Patagonia, Argentina. Cretaceous Research 27: $364-376$.

Brusatte, S.L., Nesbitt, S.J., Irmis, R.B., Butler, R.J., Benton, M.J. y Norell, M.A. 2010. The origin and early radiation of dinosaurs. Earth-Science Reviews 101: 68-100.

Calvo, J.O. y González Riga, B.J. 2003. Rinconsaurus caudamirus gen. et sp. nov., a new titanosaurid (Dinosauria, Sauropoda) from the Late Cretaceous of Patagonia, Argentina. Revista Geológica de Chile 30: 333-353.

Calvo, J.O., Porfiri, J.D., González-Riga, B.J. y Kellner, A.W. 2007a. A new Cretaceous terrestrial ecosystem from Gondwana with the description of a new sauropod dinosaur. Anais Academia Brasileira Ciencia 79: 529-41.

Calvo, J.O., González Riga, B.J. y Porfiri, J.D. 2007b. A new titanosaur sauropod from the Late Cretaceous of Neuquén, Patagonia, Argentina. Arquivos do Museu Nacional, Rio do Janeiro 65: 485-504.

Carballido, J.L. y Pol, D. 2010. The dentition of Amygdalodon patagonicus (Dinosauria: Sauropoda) and the dental evolution in basal sauropods. Comptes Rendus Palevol 9: 83-93.

Carballido, J.L. y Sander, P.M. 2013. Postcranial axial skeleton of Europasaurus holgeri (Dinosauria, Sauropoda) from the Upper Jurassic of Germany: implications for sauropod ontogeny and phylogenetic relationships of basal Macronaria. Journal of Systematic Palaeontology 3: 335-387.

Carballido, J.L., Garrido, A., Canudo, J.I. y Salgado, L. 2010. Redescription of Rayososaurus agrioensis Bonaparte (Sauropoda, Diplodocoidea), a rebbachisaurid from the early Late Cretaceous of Neuquén. Geobios 43: 493-502.

Carballido, J.L., Rauhut, O.W.M., Pol, D. y Salgado, L. 2011a. Osteology and phylogenetic relationships of Tehuelchesaurus benitezii (Dinosauria, Sauropoda) from the Upper Jurassic of Patagonia. Zoological Journal of the Linnean Society 163: 605-662.

Carballido, J.L., Pol, D., Cerda, I. y Salgado, L. 2011b. The osteology of Chubutisaurus insignis Del Corro, 1975 (Dinosauria: Neosauropoda) from the "middle" Cretaceous of central Patagonia, Ar- gentina. Journal of Vertebrate Paleontology 31: 93-110.

Casal, G.A., Allard, J.O. y Foix, N. 2015. Análisis estratigráfico y paleontológico del Cretácico superior en la Cuenca del Golfo San Jorge: nueva unidad litoestratigráfica para el Grupo Chubut. Revista de la Asociación Geológica Argentina 72: 81-99.

Casal, G., Martínez, R., Luna, M., Sciutto, J.C. y Lamanna, M. 2007. Aeolosaurus colhuehuapensis sp. nov. (Sauropoda, Titanosauria) de la Formación Bajo Barreal, Cretácico superior de Argentina. Revista Brasilera de Paleontología 10: 53-62.

Casamiquela, R.M. 1964. Sobre el hallazgo de dinosaurios triásicos en la Provincia de Santa Cruz, Argentina. Austral 35: 10-11.

Casamiquela, R.M. 1980. La presencia del género Plateosaurus (Prosauropoda) en el Triásico superior de la Formación El Tranquilo, Patagonia. $2^{\circ}$ Congreso Argentino de Paleontología y Bioestratigrafia y $1^{\circ}$ Congreso Latinoamericano de Paleontología (Buenos Aires), Actas 1: 143-156.

Cerda, I.A. y Powell, J.E. 2010. Dermal armor histology of Saltasaurus loricatus, an Upper Cretaceous sauropod dinosaur from Northwest Argentina. Acta Palaeontologica Polonica 55: 389398.

Cerda, I.A., Carabajal, A.P., Salgado, L., Coria, R.A., Reguero, M.A., Tambussi, C.P. y Molly, J.J. 2012. The first record of a sauropod dinosaur from Antarctica. Naturwissenschaften 99: 83-87.

Coria, R.A., Ortega, F., Succar, C., Currie, P. y Koppelhus, E. 2012. First record of a dicraeosaurid sauropod from the Lower Cretaceous (Valanginian) of Neuquén Basin. Ameghiniana, Suplemento Resúmenes 49: R44.

Coria, R.A., Ortega, F., Currie, P. y Cárdenas, M. 2013a. An Early Cretaceous dinosaur assemblage from the Neuquén Basin, Patagonia, Argentina. Ameghiniana, Suplemento Resúmenes 50: R13.

Coria, R.A., Filippi, L.S., Chiappe, L.M., García, R. y Arcucci, A.B. 2013 b. Overosaurus paradasorum gen. et sp. nov., a new sauropod dinosaur (Titanosauria: Lithostrotia) from the Late Cretaceous of Neuquén, Patagonia, Argentina. Zootaxa 3683: 357-376.

Del Corro, G. 1975. Un nuevo saurópodo del Cretácico superior Chubutisaurus insignis gen. et sp. nov) (Saurischia, Chubutisauridae, nov.) del Cretácico superior (Chubutiano), Chubut, Argentina. $1^{\circ}$ Congreso Argentino de Paleontologia y Bioestratigrafia (Tucumán), Actas: 229-240.

D'Emic, M.D. 2012. The early evolution of titanosauriform sauropod dinosaurs. Zoological Journal of the Linnean Society 166: 624-671.

D'Emic, M.D. y Wilson, J.A., 2011. New remains attributable to the holotype of the sauropod dinosaur Neuquensaurus australis, with implications for saltasaurine systematics. Acta Palaeontologica Polonica 56: 61-73.

Ezcurra, M.D. 2010. A new early dinosaur (Saurischia: Sauropodomorpha) from the Late Triassic of Argentina: a reassessment of dinosaur origin and phylogeny. Journal of Systematic Palaeontology 8: 371-425.

Filippi, L.S., Canudo, J.I., Salgado, L., Garrido, A.C., García, R.A., Cerda, I.A. y Otero, A. 2011a. A new sauropod titanosaur from the Plottier Formation (Upper Cretaceous) of Patagonia (Argentina). Geologica Acta 9: 1-12.

Filippi, L.S., García, R.A. y Garrido, A.C. 2011b. A new titanosaur sauropod dinosaur from the Upper Cretaceous of North Patagonia, Argentina. Acta Palaeontologica Polonica 56: 505-520.

Franco-Rosas, A.C., Salgado, L., Rosas, C.F. y Carvalho, I.S. 2004. Nuevos materiales de titanosaurios (Sauropoda) en el Cretácico superior de Mato Grosso, Brasil. Revista Brasileira de Paleontologia 7: 329-336.

Gallina, P.A. 2011. Notes on the axial skeleton of the titanosaur Bo- 
nitasaura salgadoi (Dinosauria-Sauropoda). Anais da Academia Brasileira de Ciências 83: 235-245.

Gallina, P.A. 2012. Histología ósea del titanosaurio Bonitasaura salgadoi (Dinosauria: Sauropoda) del Cretácico superior de Patagonia. Ameghiniana 49: 289-302.

Gallina, P.A. y Apesteguía, S. 2011. Cranial anatomy and phylogenetic position of the titanosaurian sauropod Bonitasaura salgadoi. Acta Palaeontologica Polonica 56: 45-60.

Gallina, P.A. y Apesteguía, S. 2015. Postcranial anatomy of Bonitasaura salgadoi (Sauropoda, Titanosauria) from the Late Cretaceous of Patagonia. Journal of Vertebrate Paleontology Doi: 10.1080/02724634.2014.924957.

Gallina, P.A., Apesteguía, S., Haluza, A., Canale, J.I. y Otero, A. $2014 a$. The sauropod fauna of the Bajada Colorada Formation (Berriasian-Valanginian), Neuquén province, Argentina. Ameghiniana, Suplemento Resúmenes 51: 11R.

Gallina, P.A., Apesteguía, S., Haluza, A. y Canale, J.I. 2014b. A Diplodocid Sauropod survivor from the Early Cretaceous of South America. PLoS ONE 9: e97128. Doi: 10.1371/journal.phone.0097128

Gallina, P.A. y Otero, A. 2015. Reassessment of Laplatasaurus araukanicus (Sauropoda: Titanosauria), from the Late Cretaceous of Patagonia, Argentina. Ameghiniana. Doi:10.5710/ AMGH.08.06.2015.2911

Galton, P.M. 1990. Basal Sauropodomorpha-Prosauropoda. En: D. Weishampel, P. Dodson y H. Osmólska (Eds.), The Dinosauria. University of California Press, Berkeley, p. 320-344.

Galton, P.M. y Upchurch, P. 2004. Prosauropoda. En: D. Weishampel, P. Dodson y H. Osmólska (Eds.), The Dinosauria 2nd edition. University of California Press, Berkeley, p. 232-258.

Garrido, A.C. 2010. Estratigrafía del Grupo Neuquén, Cretácico Superior de la Cuenca Neuquina (Argentina): nueva propuesta de ordenamiento litoestratigráfico. Revista del Museo Argentino de Ciencias Naturales 12: 121-177.

González Riga, B.J. 2003. A new titanosaur (Dinosauria, Sauropoda) from the Upper Cretaceous of Mendoza Province, Argentina. Ameghiniana 40: 155-172.

González Riga, B.J. 2005. Nuevos restos fósiles de Mendozasaurus neguyelap (Sauropoda, Titanosauria) del Cretácico tardío de Mendoza, Argentina. Ameghiniana 42: 535-548.

González Riga, B.J., Previtera, E. y Pirrone, C.A. 2009. Malarguesaurus florenciae gen. et sp. nov., a new titanosauriform (Dinosauria, Sauropoda) from the Upper Cretaceous of Mendoza, Argentina. Cretaceous Research 30: 135-148.

Haluza, A., Canale, J., Otero, A., Pérez, L. y Scanferla, C. 2012. Changes in vertebral laminae across the cervicodorsal transition of a well-preserved rebbachisaurid (Dinosauria: Sauropoda) from the Cenomanian of Patagonia, Argentina. Journal of Vertebrate Paleontology 32: 219-224.

Holwerda, F.M., Pol, D. y Rauhut, O.W.M. 2015. Using dental enamel wrinkling to define Sauropod tooth morphotypes from the Cañadón Asfalto Formation, Patagonia, Argentina. PLOS ONE 10: e0118100. Doi:10.1371/journal.pone.0118100.

Huene, F. von. 1929. Los Saurisquios y Ornitisquios del Cretáceo Argentino. Anales del Museo de la Plata 3: 1-194.

Ibiricu, L.M., Casal, G.A., Martínez, R.D., Lamanna, M.C., Luna, M. y Salgado, L. 2013. Katepensaurus goicoecheai, gen. et sp. nov., a Late Cretaceous rebbachisaurid (Sauropoda, Diplodocoidea) from central Patagonia, Argentina. Journal of Vertebrate Paleontology 33: 1351-1366.

Janensch, W. 1914. Übersicht über die Wirbeltierfauna der TendaguruSchichten, nebst einer kurzen Charakterisierung der neu aufgeführten Arten von Sauropoden. Archiv für Biontologie 3: 81-110.
Janensch, W. 1929. Material und Formengehalt der Sauropoden in der Ausbeute der Tendaguru-Expedition. Palaeontographica (Supplement 7) 2: 1-34.

Langer, M.C. 2004. Basal Saurischia. En: D. Weishampel, P. Dodson y H. Osmólska (Eds.), The Dinosauria, 2nd edition. University of California Press, Berkeley, p. 25-46.

Lavocat, R. 1954. Sur les dinosauriens du Continental Intercalaire des Kem-Kem de la Daoura. Comptes Rendus 19th International Geological Congress (Alger), Actas 1952: 65-68.

Lydekker, R.1893. The dinosaurs of Patagonia. Anales del Museo de La Plata, 2: 1-14.

Mannion, P.D. y Calvo, J.O. 2010. Anatomy of the basal titanosaur (Dinosauria, Sauropoda) Andesaurus delgadoi from the midCretaceous (Albian-early Cenomanian) Río Limay Formation, Neuquén Province, Argentina: implications for titanosaur systematics. Zoological Journal of the Linnean Society 163: 155-181.

Mannion, P.D., Upchurch, P., Barnes, R.N. y Mateus, O. 2013. Osteology of the Late Jurassic Portuguese sauropod dinosaur Lusotitan atalaiensis (Macronaria) and the evolutionary history of basal titanosauriforms. Zoological Journal of the Linnean Society 168: 98-206.

Martinelli, A.G. y Forasiepi, A.M. 2004. Late Cretaceous vertebrates from Bajo de Santa Rosa (Allen Formation), Río Negro province, Argentina, with the description of a new sauropod dinosaur (Titanosauridae). Revista Museo Argentino de Ciencias Naturales, Nueva Serie 6: 257-305.

Martínez, R.N. 2009. Adeopaposaurus mognai gen. et sp. nov. (Dinosauria: Sauropodomorpha) with comments on adaptations of basal Sauropodomorpha. Journal of Vertebrate Paleontology 29: $142-164$

Martínez, R.N. y Alcober, O.A. 2009. A basal sauropodomorph (Dinosauria: Saurischia) from the Ischigualasto Formation (Triassic, Carnian) and the early evolution of Sauropodomorpha. PLOS ONE 4: e4397. Doi: 10.1371/journal.phone.0004397

Martínez, R.N., Haro, J.A. y Apaldetti, C.G. 2012. Braincase of Panphagia protos (Dinosauria, Sauropodomorpha). Journal of Vertebrate Paleontology Memoir 12: 70-82.

Martínez, R.D., Giménez, O., Rodriguez, J., Luna, M. y Lamanna, M.C. 2004. An articulated specimen of the basal titanosaurian (Dinosauria: Sauropoda) Epachthosaurus sciuttoi from the early Late Cretaceous Bajo Barreal Formation of Chubut Province, Argentina. Journal of Vertebrate Paleontology 24: 107-120.

Martínez, R.N., Sereno, P.C., Alcober, O.A., Colombi, C.E., Renne, P.R., Montañez, I.P. y Currie, B.S. 2011. A basal dinosaur from the Dawn of the Dinosaur Era in Southwestern Pangaea. Science 331: 201-210.

McPhee, B.W., Yates, A.M., Choiniere, J.N. y Abdala, F. 2014. The complete anatomy and phylogenetic relationships of Antetonitrus ingenipes (Sauropodiformes, Dinosauria): implications for the origins of Sauropoda. Zoological Journal of the Linnean Society 171: 151-205.

Novas, F.E. 1994. New information on the systematics and postcranial skeleton of Herrerasaurus ischigualastensis (Theropoda: Herrerasauridae) from the Ischigualasto Formation (Upper Triassic) of Argentina. Journal of Vertebrate Paleontology 13: 400-423.

Otero, A. 2010. The appendicular skeleton of Neuquensaurus, a Late Cretaceous saltasaurine sauropod from Patagonia, Argentina. Acta Palaeontologica Polonica 55: 299-326.

Otero, A. y Gasparini, Z. 2014. The history of the cast skeleton of Diplodocus carnegii Hatcher, 1901, at the Museo de la Plata, Argentina. Annals of the Carnegie Museum 82: 291-304. 
Otero, A. y Pol, D. 2013. Postcranial anatomy and phylogenetic relationships of Mussaurus patagonicus (Dinosauria, Sauropodomorpha). Journal of Vertebrate Paleontology 33: 1138-1168.

Otero, A., Krupandan, E., Pol, D., Chinsamy, A. y Choiniere, J. 2015. A new basal Sauropodiform from South Africa and the phylogenetic relationships of basal sauropodomorphs. Zoological Journal of the Linnean Societt 174: 589-634.

Pérez, L.M., Otero, A., Apesteguía, S. y Gallina, P.A. 2009. Estratigrafía y análisis tafonómico de Bonitasaura salgadoi Apesteguía, en el sitio "La Bonita" (Cretácico superior, Río Negro, Argentina). Revista del Museo Argentino de Ciencias Naturales 11: 39-48.

Pol, D. y Powell, J.E. 2007. New information on Lessemsaurus sauropoides (Dinosauria: Sauropodomorpha) from the Upper Triassic of Argentina. Special Papers in Palaeontology 77: 223-243.

Pol, D., Garrido, A. y Cerda, I.A. 2011. A new Sauropodomorph dinosaur from the Early Jurassic of Patagonia and the origin and evolution of the Sauropod-type sacrum. PLOS ONE 6: e14572. Doi:10.1371/journal.pone.0014572.

Powell, J.E. 1986. [Revisión de los Titanosaurios de América del Sur. Tesis Doctoral, Universidad Nacional de Tucumán, Argentina, 340 p. Inédita.].

Powell, J.E. 1992. Osteología de Saltasaurus loricatus (SauropodaTitanosauridae) del Cretácico superior del Noroeste Argentino. En: J.L. Sanz y A.D. Buscalioni (Eds.), Los Dinosaurios y su Entorno Biótico. Actas del Segundo Curso de Paleontología de Cuenca. Instituto "Juan de Valdéz", Cuenca, p. 165-230.

Powell, J.E. 2003. Revision of South American titanosaurid dinosaurs: paleobiological, palaeobiogeographical and phylogenetic aspects. Record of the Queen Victoria Museum Launceston 11: $1-173$.

Rauhut, O.W.M. 2002. Los dinosaurios de la Formación Cañadón Asfalto. Ameghiniana, Suplemento Resúmenes 39: 15R-16R.

Rauhut, O.W.M. 2003. Revision of Amygdalodon patagonicus Cabrera, 1947 (Dinosauria Sauropoda). Mitteilungen des Museums für Naturkunde Berlin, Geowiss. Reihe 6: 173-181.

Rich, T.H., Vickers-Rich, P., Gimenez, O., Cúneo, R., Puerta, P. y Vacca, R. 1999. A new sauropod dinosaur from Chubut Province, Argentina. National Science Museum Monographs 15: 61-84.

Salgado, L. 1993. Comments on Chubutisaurus insignis Del Corro. Ameghiniana 30: 265-270.

Salgado, L. y Bonaparte, J.F. 2007. Sauropodomorpha. En: Z. Gasparini, L. Salgado y R.A. Coria (Eds.), Patagonian Mesozoic Reptiles. Indiana University Press, Bloomington, p. 188-228.

Salgado, L. y Coria, R.A. 2009. Barrosasaurus casamiquelai gen. et sp. nov., a new titanosaur (Dinosauria, Sauropoda) from the Anacleto Formation (Late Cretaceous: early Campanian) of Sierra Barrosa (Neuquén, Argentina). Zootaxa 2222: 1-16.

Salgado, L., Coria, R.A. y Calvo, J.O. 1997a. Evolution of titanosaurid sauropods I: Phylogenetic analysis based on the postcranial evidence. Ameghiniana 34: 3-32.

Salgado, L., Coria, R.A. y Calvo, J.O. 1997b. Presencia del género Aeolosaurus (Sauropoda, Titanosauridae) en la Formación Los Alamitos, Cretácico Superior de la provincia de Río Negro, Argentina. Revista Universidade Guarulhos, Geociencias 2: 44-46.

Salgado, L., Apesteguía, S. y Heredia, S.E. 2005. A new specimen of Neuquensaurus australis, a Late Cretaceous Saltasaurinae titanosaur from North Patagonia. Journal of Vertebrate Paleontology 25: 623-634.

Salgado, L., Gallina, P.A. y Paulina Carabajal, A. 2014. Redescription of Bonatitan reigi (Sauropoda: Titanosauria), from the Campanian-Maastrichtian of the Río Negro Province (Argentina). Historical Biology 27: 525-548.
Salgado, L., Garrido, A., Cocca, S. y Cocca, J.R. 2004. Lower Cretaceous rebbachisaurid sauropods frorn Cerro Aguada del León (Lohan Cura Formation), Neuquén Province, northwestern Patagonia, Argentina. Journal of Vertebrate Paleontology 24: 903-912.

Sanz, J.L. 2007. Cazadores de dragones. Historia del descubrimiento e investigación de los dinosaurios. Editorial Ariel, Barcelona, 420 p.

Sereno, P.C. 1999. The evolution of dinosaurs. Science 284: 21372147.

Sereno, P.C. 2006. The phylogenetic relationships of early dinosaurs: a comparative report. Historical Biology 19: 145-155.

Sereno, P.C. 2007. Basal Sauropodomorpha: historical and recent phylogenetic hypotheses, with comments on Ammosaurus major (Marsh, 1889). Special Papers in Palaeontology 77: 261-289.

Sereno, P.C., Martínez, R.N. y Alcober, O.A. 2012. Osteology of Eoraptor lunensis (Dinosauria, Sauropodomorpha). Society of Vertebrate Paleontology Memoir 12: 83-179.

Smith, N.D. y Pol, D. 2007. Anatomy of a basal sauropodomorph dinosaur from the Early Jurassic Hanson Formation of Antarctica. Acta Palaeontologica Polonica 52: 657-674.

Smith, R.M.H., Marsicano, C.A., Pol, D. y Mancuso, A.C. 2014. Ichnology of sauropodomorph nests from Patagonia indicates Early Jurassic origin of herd-living and herding site fidelity. $4^{\text {th }}$ International Paleontological Congress (Mendoza), Actas: 227.

Tykoski, R.S. 2005. [Anatomy, ontogeny, and phylogeny of coelophysoid theropods. Tesis Doctoral. University of Texas, Austin, Texas, 553 p. Inédita.].

Upchurch, P., Barrett, P.M. y Dodson, P. 2004. Sauropoda. En: D.B. Weishampel, P. Dodson y H. Osmólska (Eds.), The Dinosauria 2nd edition. University California Press, Berkeley, p. 259-322.

Upchurch, P., Barrett, P.M. y Galton, P.M. 2007. A phylogenetic analysis of basal sauropodomorph relationships: implications for the origin of sauropod dinosaurs. Special Papers in Palaeontology 77: 57-90.

Varela, A.N., Veiga, G.D. y Poiré, D.G. 2012a. Sequence stratigraphic analysis of Cenomanian greenhouse palaeosols: A case study from southern Patagonia, Argentina. Sedimentary Geology 271272: 67-82.

Varela, A.N., Poiré, D.G., Martin, T., Gerdes, A., Goin, F.J., Gelfo, J.N. y Hoffmann, S. 2012b. U-Pb zircon constraints on the age of the Cretaceous Mata Amarilla Formation, Southern Patagonia, Argentina: its relationship with the evolution of the Austral Basin. Andean Geology 39: 359-379.

Whitlock, J.A. 2011. A phylogenetic analysis of Diplodocoidea (Saurischia: Sauropoda). Zoological Journal of the Linnean Society 161: 872-915.

Wilson, J.A. 2002. Sauropod dinosaur phylogeny: critique and cladistic analysis. Zoological Journal of the Linnean Society 136: 217-276.

Wilson, J.A. y Sereno, P.C. 1998. Early evolution and higher-level phylogeny of sauropod dinosaurs. Society of Vertebrate Paleontology Memoir 5: 1-68.

Wilson, J.A. y Carrano, M.T. 1999. Titanosaurs and the origin of "wide-gauge" trackways: a biomechanical and systematic perspective on sauropod locomotion. Paleobiology 25: 252-267.

Wilson, J.A. y Upchurch, P. 2003. A revision of Titanosaurus Lydekker (Dinosauria - Sauropoda), the first dinosaur genus with a 'Gondwanan' distribution. Journal of Systematic Palaeontology 1 : 125-160.

Yates, A.M. 2007a. The first complete skull of the Triassic dinosaur Melanorosaurus Haughton (Sauropodomorpha: Anchisauria). Special Papers in Palaeontology 77: 9-55. 
Yates, A.M. 2007b. Solving a dinosaurian puzzle: the identity of Aliwalia rex Galton. Historical Biology 19: 93-123.

Yates, A.M. y Kitching, J.W. 2003. The earliest known sauropod dinosaur and the first steps towards sauropod locomotion. Proceedings of the Royal Society of London B 270: 1753-1758.

Zurriaguz, V. y Powell, J.E. 2015. New contributions to the presacral osteology of Saltasaurus loricatus (Sauropoda, Titanosauria) from the Upper Cretaceous of northern Argentina. Cretaceous Research 54: 283-300.

Recibido: 4 de junio de 2015

Aceptado: 12 de agosto de 2015 
ANEXo 1 - Resumen del registro de Sauropodomorpha en la Argentina.

\begin{tabular}{|c|c|c|c|c|}
\hline $\begin{array}{l}\text { Procedencia estratigráfica } \\
\text { (Formación) }\end{array}$ & Taxón & $\begin{array}{l}\text { Procedencia geográfica } \\
\text { (Provincia) }\end{array}$ & Edad (piso) & Autor \\
\hline Ischigualasto & Eoraptor lunensis & San Juan & Carniense medio & Sereno et al., 1993 \\
\hline Ischigualasto & Panphagia protos & San Juan & Carniense medio & Martínez y Alcober, 2009 \\
\hline Ischigualasto & Chromogisaurus novasi & San Juan & Carniense medio & Ezcurra, 2010 \\
\hline Los Colorados & Riojasaurus incertus & La Rioja & Noriense/Rhaetiense & Bonaparte, 1967 \\
\hline Los Colorados & Coloradisaurus brevis & La Rioja & Noriense/Rhaetiense & Bonaparte, 1978 \\
\hline Los Colorados & Lessemsaurus sauropoides & La Rioja & Noriense-Rhaetiense & Bonaparte, 1999a \\
\hline Laguna Colorada & Mussaurus patagonicus & Santa Cruz & Sinemuriense & Bonaparte y Vince, 1979 \\
\hline Quebrada del Barro & Leyesaurus marayensis & San Juan & Jurásico Inferior* & Apaldetti et al., 2011 \\
\hline Cañón del Colorado & Adeopapposaurus mognai & San Juan & Jurásico Inferior* & Martínez, 2009 \\
\hline Las Leoneras & Leonerasaurus taquetrensis & Chubut & Toarciense? & Polet al., 2011 \\
\hline Cerro Carnerero & Amygdalodon patagonicus & Chubut & Toarciense/Bajociense & Cabrera, 1947 \\
\hline Cañadón Asfalto & Patagosaurus fariasi & Chubut & Aaleniense/Batoniense & Bonaparte, 1979 \\
\hline Cañadón Asfalto & Volkheimeria chubutensis & Chubut & Aaleniense/Batoniense & Bonaparte, 1979 \\
\hline Cañadón Calcáreo & Brachytrachelopan mesai & Chubut & Titoniense & Rauhut et al., 2005 \\
\hline Cañadón Calcáreo & Tehuelchesaurus benitezi & Chubut & Kimmeridgiense/Titoniense & Rich et al., 1999 \\
\hline Bajada Colorada & Leinkupal laticauda & Neuquén & Berriasiense/Valanginiense & Gallina et al., 2014 \\
\hline Rayoso & Rayososaurus agrioensis & Neuquén & Aptiense & Bonaparte, 1996 \\
\hline La Amarga & Amargasaurus cazaui & Neuquén & Barremiense & Salgado y Bonaparte, 1991 \\
\hline La Amarga & Zapalasaurus bonapartei & Neuquén & Barremiense/Aptiense & Salgado et al., 2006 \\
\hline Lohan Cura & Ligabuesaurus leanzai & Neuquén & Aptiense & Bonaparte et al., 2006 \\
\hline Lohan Cura & Agustinia ligabuei & Neuquén & Aptiense & Bonaparte, $1999 b$ \\
\hline Lohan Cura & Comahuesaurus windhauseni & Neuquén & Aptiense/Albiense & Carballido et al., 2012 \\
\hline Candeleros & Limaysaurus tessonei & Neuquén & Cenomaniense Inferior & (Calvo y Salgado, 1995) \\
\hline Candeleros & Andesaurus delgadoi & Neuquén & Cenomaniense Inferior & Calvo y Bonaparte, 1991 \\
\hline Huincul & Cathartesaura anaerobica & Río Negro & Cenomaniense Superior & Gallina y Apesteguía, 2005 \\
\hline Huincul & Argentinosaurus huinculensis & Neuquén & Cenomaniense Superior & Bonaparte y Coria, 1993 \\
\hline Cerro Barcino & Chubutisaurus insignis & Chubut & Cenomaniense & del Corro, 1975 \\
\hline Bajo Barreal & Katepensaurus goicoecheai & Chubut & Cenomaniense/Turoniense & Ibiricu et al., 2013 \\
\hline Bajo Barreal & Epachthosaurus sciuttoi & Chubut & Cenomaniense/Turoniense & Powell, 1990 \\
\hline Bajo Barreal & Drusilasaura deseadensis & Santa Cruz & Cenomaniense/Turoniense & Navarrete et al., 2011 \\
\hline Río Neuquén & Mendozasaurus neguyelap & Mendoza & Turoniense/Coniaciense & González Riga, 2003 \\
\hline Portezuelo & Futalognkosaurus dukei & Neuquén & Turoniense/Coniaciense & Calvo et al., 2007 \\
\hline Portezuelo & Malarguesaurus florenciae & Mendoza & Turoniense/Coniaciense & González Riga et al., 2009 \\
\hline Portezuelo & Muyelensaurus pecheni & Neuquén & Turoniense/Coniaciense & Calvo et al., 2007 \\
\hline Río Neuquén & Rinconsaurus caudamirus & Neuquén & Turoniense/Coniaciense & Calvo y González Riga, 2003 \\
\hline
\end{tabular}




\begin{tabular}{|c|c|c|c|c|}
\hline \multicolumn{5}{|l|}{ Anexo 1 - Continuación. } \\
\hline $\begin{array}{l}\text { Procedencia estratigráfica } \\
\text { (Formación) }\end{array}$ & Taxón & $\begin{array}{l}\text { Procedencia geográfica } \\
\text { (Provincia) }\end{array}$ & Edad (piso) & Autor \\
\hline Colhue Huapi & Argyrosaurus superbus & Chubut & Coniaciense/Maastrichtiense & Lydekker, 1893 \\
\hline Colhue Huapi & Elaltitan lilloi & Chubut & Coniaciense/Maastrichtiense & Mannion y Otero, 2012 \\
\hline Colhue Huapi & Aeolosaurus colhuehuapensis & Chubut & Coniaciense/Maastrichtiense & Casal et al., 2007 \\
\hline Plottier & $\begin{array}{l}\text { Petrobrasaurus } \\
\text { puestohernandezi }\end{array}$ & Neuquén & Santoniense & Filippi et al., 2011 \\
\hline Bajo de La Carpa & Bonitasaura salgadoi & Río Negro & Santoniense & Apesteguía, 2004 \\
\hline Anacleto & Neuquensaurus australis & Neuquén & Campaniense & (Huene, 1929) \\
\hline Anacleto & Laplatasaurus araukanicus & Río Negro & Campaniense & Huene, 1929 \\
\hline Anacleto & Pellegrinisaurus powelli & Río Negro & Campaniense & Salgado, 1990 \\
\hline Anacleto & Barrosasaurus casamiquelai & Neuquén & Campaniense & Salgado y Coria, 2009 \\
\hline Anacleto & Pitekunsaurus macayai & Neuquén & Campaniense & Filippi y Garrido, 2008 \\
\hline Anacleto & Narambuenatitan palomoi & Neuquén & Campaniense & Filippi et al., 2011 \\
\hline Anacleto & Overosaurus paradasorum & Neuquén & Campaniense & Coria et al., 2013 \\
\hline Cerro Fortaleza & Dreadgnouthus schrani & Santa Cruz & Campaniense & Lacovara et al., 2014 \\
\hline Pari Aike & Puertasaurus reuilli & Santa Cruz & Maastrichtiense & Novas et al., 2005 \\
\hline Angostura Colorada & Aeolosaurus rionegrinus & Río Negro & Campaniense/Maastrichtiense & Powell, 1986 \\
\hline Allen & Antarctosaurus wichmannianus & Río Negro & Campaniense/Maastrichtiense & Huene, 1929 \\
\hline Allen & Rocasaurus muniozi & Río Negro & Campaniense/Maastrichtiense & Salgado y Azpilicueta, 2000 \\
\hline Allen & Bonatitan reigi & Río Negro & Campaniense/Maastrichtiense & Martinelli y Forasiepi, 2004 \\
\hline Allen & Panamericansaurus schroederi & Neuquén & Campaniense/Maastrichtiense & Calvo y Porfiri, 2010 \\
\hline Lecho & Saltasaurus loricatus & Salta & Campaniense/Maastrichtiense & Bonaparte y Powell, 1980 \\
\hline
\end{tabular}

